Electronic Journal of Statistics

Vol. 14 (2020) 3192-3229

ISSN: 1935-7524

https://doi.org/10.1214/20-EJS1738

\title{
Asymptotic properties for the parameter estimation in Ornstein-Uhlenbeck process with discrete observations
}

\author{
Hui Jiang* ${ }^{* \dagger}$ and Hui Liu \\ Department of Mathematics, \\ Nanjing University of Aeronautics and Astronautics, \\ Nanjing 211106, China \\ e-mail: huijiang@nuaa.edu.cn; lhui56@163.com \\ Youzhou Zhou ${ }^{\ddagger}$ \\ Department of Pure Mathematics, \\ $X i$ 'an Jiaotong-Liverpool University, \\ Suzhou 215123, China \\ e-mail: youzhou.zhou@xjtlu.edu.cn
}

\begin{abstract}
In this paper, under discrete observations, we study Cramértype moderate deviations (extended central limit theorem) for parameter estimation in Ornstein-Uhlenbeck process. Our results contain both stationary and explosive cases. For applications, we propose test statistics which can be used to construct rejection regions in the hypothesis testing for the drift coefficient, and the corresponding probability of type II error tends to zero exponentially. Simulation study shows that our test statistics have good finite-sample performances both in size and power. The main methods include the deviation inequalities for multiple Wiener-Itô integrals, as well as the asymptotic analysis techniques.
\end{abstract}

MSC2020 subject classifications: $62 \mathrm{~N} 02,60 \mathrm{~F} 10,60 \mathrm{G} 22$.

Keywords and phrases: Ornstein-Uhlenbeck process, discrete observations, moderate deviation principle, multiple Wiener-Itô integrals.

Received January 2020

\section{Introduction and main results}

\subsection{Introduction}

The Ornstein-Uhlenbeck $(\mathrm{O}-\mathrm{U})$ process is defined as

$$
d X_{t}=\theta X_{t} d t+d W_{t}, \quad X_{0}=0, \quad t \geq 0
$$

${ }^{*}$ Corresponding Author.

${ }^{\dagger}$ Hui Jiang is supported by National Natural Science Foundation of China (No. 11771209, 11971227).

${ }^{\ddagger}$ Youzhou Zhou is supported by National Natural Science Foundation of China (No. 11701570). 
where $W=\left\{W_{t}, t \geq 0\right\}$ is a standard Brownian motion and $\theta$ is an unknown parameter. It is a reduced Vasicek model ([8], [9], [45]) which is often used to describe term structure. This model can successfully characterize mean reversion property of short interest rate. The parameter $\theta$ indicates the reverting rate. In model (1.1), the average interest rate is 0 . When $\theta<0$, short rate $X_{t}$ will always be pulled back to its average level if it is away from 0 . However, the short rate $X_{t}$ will deviate from average level much more drastically when $\theta>0$. Therefore, $\theta<0$ is called stationary case and $\theta>0$ is called explosive case. The trivial case $\theta=0$ indicates that the short rate $X_{t}$ is completely random (in this case, the O-U process is Brownian motion).

It is of great importance to estimate parameter $\theta$ in financial market. Also interest rate is usually regularly reported, so we can consider the parameter estimation under discrete observations. Let $\left\{t_{i} \mid t_{i}=i \Delta, 0 \leq i \leq n, \Delta>0\right\}$ be sampling times and $\left\{X_{i} \mid X_{i}=X_{t_{i}}, 0 \leq i \leq n\right\}$ be observed samples. Kasonga ([28]) proposed the following estimator

$$
\hat{\theta}_{n, \Delta}= \begin{cases}\frac{1}{\Delta} \log \left(\frac{\sum_{i=1}^{n} X_{i} X_{i-1}}{\sum_{i=1}^{n} X_{i-1}^{2}}\right), & \text { if } \frac{\sum_{i=1}^{n} X_{i} X_{i-1}}{\sum_{i=1}^{n} X_{i-1}^{2}}>0 \\ 0, & \text { otherwise. }\end{cases}
$$

He also studied the weak consistency property for $\hat{\theta}_{n, \Delta}$ in ([28]). In this article, we assume

$$
(H): \Delta \rightarrow 0, \quad n \Delta \rightarrow \infty, \quad n \rightarrow \infty .
$$

Thus, we are dealing with high frequency observations. Shimizu ([43]) obtained the asymptotic distribution of Kasonga's estimator $\hat{\theta}_{n, \Delta}$ under this high frequency observations. To be explicit, for explosive case, as $\Delta \rightarrow 0, n \rightarrow \infty$ (Theorem 2 in page 198 of [43]),

$$
\frac{e^{\theta n \Delta}}{2 \theta}\left(\hat{\theta}_{n, \Delta}-\theta\right) \stackrel{\mathcal{L}}{\rightarrow} \frac{\nu}{\eta},
$$

where $\nu$ and $\eta$ are two independent standard normal variables; while for stationary case, as $\Delta \rightarrow 0, n \rightarrow \infty$ (Corollary 1 in page 199 of [43]),

$$
\sqrt{\frac{n \Delta}{-2 \theta}}\left(\hat{\theta}_{n, \Delta}-\theta\right) \stackrel{\mathcal{L}}{\rightarrow} \nu .
$$

Moreover, there are also some research works on maximum likelihood estimator of $\theta$ in continuous observation case. For stationary case, one can refer to $([4],[6],[16])$ for the large deviations, and ([17], [18], [20], [26]) for Cramértype moderate deviations, and ([1], [11], [14], [15]) for the Berry-Esseen bounds. In explosive case, Bercu et al. ([5]), Bercu and Richou ([7]) obtained the large deviations for the maximum likelihood estimator of $\theta$, while Jiang and Zhang ([27]) considered the Cramér-type moderate deviations. More study on the statistical inferences for Vasicek type processes can be found in Azmoodeh and Morlanes ([2]), Barboza and Viens ([3]), Dietz and Kutoyants ([10]), El Onsy et al. ([12]), Hu and Nualart ([22]), Hu et al. ([23], [24]), Nourdin and Tran ([37]), 
Tudor and Viens ([44]), Xiao et al. ([46], [47]), Zang and Zhang ([48], [49]) and the references therein.

In the discrete observation case, Shimizu ([42]) proved the asymptotic normality for the maximum likelihood estimator of $\theta$ in the stationary case. For more references, one can see Gobet [21], Kessler [30], Kutoyants [33], Prakasa Rao ([40]). For the explosive diffusion process, there are only literatures about consistency and asymptotic distribution of estimators, such as ([13], [25], [29], [31], [32] and the references therein). However, Cramér-type moderate deviations are still missing in both stationary case and explosive case.

In this paper, our motivation is to study the Cramér-type moderate deviations (so-called extended central limit theorem) for Kasonga's estimator $\hat{\theta}_{n, \Delta}$, and we handle both stationary case and explosive case. By Cramér-type moderate deviation, we mean there exists sequence $\alpha_{n}$ and $\beta_{n}$ such that for every $\rho>0$

$$
\frac{1}{1-F(x)} P\left(\alpha_{n}\left(\hat{\theta}_{n, \Delta}-\theta\right) \geq x\right) \rightarrow 1 \quad \forall 0 \leq x \leq \rho \beta_{n},
$$

where $F(x)$ is the limiting fluctuation distribution of $\hat{\theta}_{n, \Delta}$. Therefore, Cramértype moderate deviation provides a nice comparison between tail probability of estimator and limiting fluctuation distribution. Especially one always need to take care of these issues in hypothesis testing. We benefit a lot from Gao et al. ([19]).

\subsection{Main results}

In the following, we will state our main results in explosive case and stationary cases as well.

\subsubsection{Explosive case: $\theta>0$}

We denote $\mathbb{C}=\frac{\nu}{\eta}$, where $\nu$ and $\eta$ are two independent standard normal variables. Then $\mathbb{C}$ follows standard Cauchy distribution. Let $F_{\mathbb{C}}(x)$ be its cumulative distribution function. Let $\beta_{n, \Delta}$ be positive numbers and satisfy

$$
\beta_{n, \Delta} \rightarrow \infty, \quad \frac{n \Delta \beta_{n, \Delta}^{8}\left(\log \beta_{n, \Delta}\right)^{4}}{e^{2 \theta n \Delta}} \rightarrow 0
$$

Firstly, we show that the event $\left\{\frac{\sum_{i=1}^{n} X_{i} X_{i-1}}{\sum_{i=1}^{n} X_{i-1}^{2}} \leq 0\right\}$ is negligible in the sense of Cramér-type moderate deviation.

Theorem 1.1. Under assumption $(H)$ and (1.5), we have

$$
\lim _{\Delta \rightarrow 0, n \rightarrow \infty} \beta_{n, \Delta} P\left(\frac{\sum_{i=1}^{n} X_{i} X_{i-1}}{\sum_{i=1}^{n} X_{i-1}^{2}} \leq 0\right)=0 .
$$

Now, we state the Cramér-type moderate deviation for $\hat{\theta}_{n, \Delta}$. 
Theorem 1.2. Under assumption $(H)$ and (1.5), for each $\rho>0$, and for all $0 \leq x \leq \rho \beta_{n, \Delta}$,

$$
\lim _{\Delta \rightarrow 0, n \rightarrow \infty} \frac{1}{1-F_{\mathbb{C}}(x)} P\left(\frac{\Delta e^{\theta(n-1) \Delta}}{1-e^{-2 \theta \Delta}}\left(\hat{\theta}_{n, \Delta}-\theta\right) \geq x\right)=1
$$

and

$$
\lim _{\Delta \rightarrow 0, n \rightarrow \infty} \frac{1}{F_{\mathbb{C}}(-x)} P\left(\frac{\Delta e^{\theta(n-1) \Delta}}{1-e^{-2 \theta \Delta}}\left(\hat{\theta}_{n, \Delta}-\theta\right) \leq-x\right)=1 .
$$

Under assumption $(H)$, one can easily show that

$$
\lim _{\Delta \rightarrow 0, n \rightarrow \infty} \frac{\frac{\Delta e^{\theta(n-1) \Delta}}{1-e^{-2 \theta \Delta}}}{\frac{e^{\theta n \Delta}}{2 \theta}}=1 .
$$

Then we have the following corollary.

Corollary 1.1. Under assumption $(H)$, there exists $\beta_{n, \Delta}$, satisfying (1.5) and $\lim _{\Delta \rightarrow 0, n \rightarrow \infty} \Delta \beta_{n, \Delta}=0$, then, for each $\rho>0$, uniformly for $0 \leq x \leq \rho \beta_{n, \Delta}$,

$$
\lim _{\Delta \rightarrow 0, n \rightarrow \infty} \frac{1}{1-F_{\mathbb{C}}(x)} P\left(\frac{e^{\theta n \Delta}}{2 \theta}\left(\hat{\theta}_{n, \Delta}-\theta\right) \geq x\right)=1
$$

and

$$
\lim _{\Delta \rightarrow 0, n \rightarrow \infty} \frac{1}{F_{\mathbb{C}}(-x)} P\left(\frac{e^{\theta n \Delta}}{2 \theta}\left(\hat{\theta}_{n, \Delta}-\theta\right) \leq-x\right)=1 .
$$

However, the above results can not be directly applied in statistical inference, because the scaling factor of fluctuation limit depends on the unknown parameter $\theta$. To circumvent this difficulty, one usually replace the unknown parameter by its estimation $\hat{\theta}_{n, \Delta}$. By consistency property of estimator $\hat{\theta}_{n, \Delta}$, one can also show a self-normalized version of fluctuation limiting theorem

$$
\frac{\Delta e^{\hat{\theta}_{n, \Delta}(n-1) \Delta}}{1-e^{-2 \hat{\theta}_{n, \Delta} \Delta}}\left(\hat{\theta}_{n, \Delta}-\theta\right) \stackrel{\mathcal{L}}{\rightarrow} \frac{\nu}{\eta} .
$$

Then, the associated Cramér-type moderate deviations can be stated as follows.

Theorem 1.3. Under assumption $(H)$, there exists $\beta_{n, \Delta}$ satisfying (1.5) such that, for each $\rho>0$, uniformly for $0 \leq x \leq \rho \beta_{n, \Delta}$,

$$
\lim _{\Delta \rightarrow 0, n \rightarrow \infty} \frac{1}{1-F_{\mathbb{C}}(x)} P\left(\frac{\Delta e^{\hat{\theta}_{n, \Delta}(n-1) \Delta}}{1-e^{-2 \hat{\theta}_{n, \Delta} \Delta}}\left(\hat{\theta}_{n, \Delta}-\theta\right) \geq x\right)=1
$$

and

$$
\lim _{\Delta \rightarrow 0, n \rightarrow \infty} \frac{1}{F_{\mathbb{C}}(-x)} P\left(\frac{\Delta e^{\hat{\theta}_{n, \Delta}(n-1) \Delta}}{1-e^{-2 \hat{\theta}_{n, \Delta} \Delta}}\left(\hat{\theta}_{n, \Delta}-\theta\right) \leq-x\right)=1 .
$$


Corollary 1.2. Under assumption $(H)$, let $b_{n, \Delta}$ be positive numbers, satisfying

$$
b_{n, \Delta} \rightarrow \infty, \quad \frac{b_{n, \Delta}}{n \Delta} \rightarrow 0
$$

Then the families

$$
\left\{\left|\frac{e^{\theta n \Delta}}{2 \theta}\left(\hat{\theta}_{n, \Delta}-\theta\right)\right|^{\frac{1}{b_{n, \Delta}}}, n \geq 1, \Delta>0\right\}
$$

and

$$
\left\{\left|\frac{\Delta e^{\hat{\theta}_{n, \Delta}(n-1) \Delta}}{1-e^{-2 \hat{\theta}_{n, \Delta} \Delta}}\left(\hat{\theta}_{n, \Delta}-\theta\right)\right|^{\frac{1}{b_{n, \Delta}}}, n \geq 1, \Delta>0\right\}
$$

satisfy the same large deviations with the speed $b_{n, \Delta}$ and rate function

$$
I(x)= \begin{cases}\log x, & \text { if } x \geq 1 \\ 0, & \text { if } 0<x<1 .\end{cases}
$$

To be explicit, for any $x \geq 0$, we have

$$
\lim _{\Delta \rightarrow 0, n \rightarrow \infty} \frac{1}{b_{n, \Delta}} \log P\left(\left|\frac{e^{\theta n \Delta}}{2 \theta}\left(\hat{\theta}_{n, \Delta}-\theta\right)\right|^{\frac{1}{b_{n, \Delta}}} \geq x\right)=-I(x)
$$

and

$$
\lim _{\Delta \rightarrow 0, n \rightarrow \infty} \frac{1}{b_{n, \Delta}} \log P\left(\left|\frac{\Delta e^{\hat{\theta}_{n, \Delta}(n-1) \Delta}}{1-e^{-2 \hat{\theta}_{n, \Delta} \Delta}}\left(\hat{\theta}_{n, \Delta}-\theta\right)\right|^{\frac{1}{b_{n, \Delta}}} \geq x\right)=-I(x) .
$$

\subsubsection{Ergodic case: $\theta<0$}

In this subsection, we will strengthen the assumption $(H)$ by

$$
\left(H^{\prime}\right): \Delta \rightarrow 0, \quad n \Delta \rightarrow \infty, \quad n \Delta^{4}(\log n \Delta)^{6} \rightarrow 0, \quad n \rightarrow \infty .
$$

Under assumption $\left(H^{\prime}\right)$, let $\lambda_{n, \Delta}$ be positive numbers, satisfying

$$
\lambda_{n, \Delta} \rightarrow \infty, \quad \frac{\lambda_{n, \Delta}}{(n \Delta)^{1 / 12}} \rightarrow 0 .
$$

Similar to the explosive case, we first show the event $\left\{\frac{\sum_{i=1}^{n} X_{i} X_{i-1}}{\sum_{i=1}^{n} X_{i-1}^{2}} \leq 0\right\}$ is negligible in the sense of Cramér-type moderate deviation.

Theorem 1.4. Under assumption $\left(H^{\prime}\right)$ and (1.16), we have

$$
\lim _{\Delta \rightarrow 0, n \rightarrow \infty} \frac{1}{\lambda_{n, \Delta}^{2}} \log P\left(\frac{\sum_{i=1}^{n} X_{i} X_{i-1}}{\sum_{i=1}^{n} X_{i-1}^{2}} \leq 0\right)=-\infty .
$$


Theorem 1.5. Then, for each $\rho>0$, uniformly for $0 \leq x \leq \rho \lambda_{n, \Delta}$,

$$
\frac{1}{1-\Phi(x)} P\left(\sqrt{\frac{n \Delta}{-2 \theta}}\left(\hat{\theta}_{n, \Delta}-\theta\right) \geq x\right) \rightarrow 1
$$

and

$$
\frac{1}{\Phi(-x)} P\left(\sqrt{\frac{n \Delta}{-2 \theta}}\left(\hat{\theta}_{n, \Delta}-\theta\right) \leq-x\right) \rightarrow 1
$$

where $\Phi(x)$ is the standard normal distribution function.

Similarly, to apply the above result in statistical inference, we need to replace the unknown parameter $\theta$ by its estimation. Therefore, we consider a self-normalized version of $\hat{\theta}_{n, \Delta}$. To be explicit, from (1.4) and the fact that $\hat{\theta}_{n, \Delta}$ is a consistent estimator of $\theta$, we can derive the following result immediately,

$$
\sqrt{\frac{n \Delta}{-2 \hat{\theta}_{n, \Delta}}}\left(\hat{\theta}_{n, \Delta}-\theta\right) \stackrel{\mathcal{L}}{\rightarrow} \nu
$$

Then, the associated Cramér-type moderate deviation can be stated as follows.

Theorem 1.6. Under assumption $\left(H^{\prime}\right)$, let $\lambda_{n, \Delta}$ satisfy (1.16). Then, for each $\rho>0$, uniformly for $0 \leq x \leq \rho \lambda_{n, \Delta}$,

$$
\frac{1}{1-\Phi(x)} P\left(\sqrt{\frac{n \Delta}{-2 \hat{\theta}_{n, \Delta}}}\left(\hat{\theta}_{n, \Delta}-\theta\right) \geq x\right) \rightarrow 1
$$

and

$$
\frac{1}{\Phi(-x)} P\left(\sqrt{\frac{n \Delta}{-2 \hat{\theta}_{n, \Delta}}}\left(\hat{\theta}_{n, \Delta}-\theta\right) \leq-x\right) \rightarrow 1 .
$$

Corollary 1.3. Under assumption $\left(H^{\prime}\right)$, let $\lambda_{n, \Delta}$ satisfy (1.16), the following families

$$
\left\{\frac{1}{\lambda_{n, \Delta}} \sqrt{\frac{n \Delta}{-2 \theta}}\left(\hat{\theta}_{n, \Delta}-\theta\right), n \geq 1, \Delta>0\right\}
$$

and

$$
\left\{\frac{1}{\lambda_{n, \Delta}} \sqrt{\frac{n \Delta}{-2 \hat{\theta}_{n, \Delta}}}\left(\hat{\theta}_{n, \Delta}-\theta\right), n \geq 1, \Delta>0\right\}
$$

satisfy the same large deviation with speed $\lambda_{n, \Delta}^{2}$ and the rate function $\Lambda(x)=$ $x^{2} / 2, x \in \mathbb{R}$.

The remaining part of this paper is organized as follows. In Section 2, we apply our results to confidence interval and hypothesis testing problem. Simulation study shows that our test statistics have good finite-sample performances both in size and power. Then, the proofs of our main results will be presented 
in Section 3. Two key technical propositions will be proved in Section 4 by using deviation inequalities for multiple Wiener-Itô integrals and the asymptotic analysis techniques. Finally, we introduce the Cramér-type moderate deviations for multiple Wiener-Itô integrals in Appendix section. Throughout this paper, $C, C_{1}, C_{2}$ depending only on $\theta$, denote positive constants whose values can differ at different places.

\section{Statistical applications}

\subsection{Explosive case: $\theta>0$}

(I) Confidence interval. An application of (1.10) yields the following approximate $(1-\alpha)(0<\alpha<1)$ confidence interval for $\theta$ :

$$
\left(\hat{\theta}_{n, \Delta}-\frac{1-e^{-2 \hat{\theta}_{n, \Delta} \Delta}}{\Delta e^{\hat{\theta}_{n, \Delta}(n-1) \Delta}} t_{\alpha / 2}(1), \hat{\theta}_{n, \Delta}+\frac{1-e^{-2 \hat{\theta}_{n, \Delta} \Delta}}{\Delta e^{\hat{\theta}_{n, \Delta}(n-1) \Delta}} t_{\alpha / 2}(1)\right)
$$

where $t_{\alpha / 2}(1)$ is the upper $\alpha / 2$-quantile of t-distribution with 1 degree of freedom. To assess the performance of above confidence interval, we need to use the Berry-Esseen bound for the estimator $\hat{\theta}_{n, \Delta}$. To the best of our knowledge, the Berry-Esseen bound for the explosive case remains open, which will be studied in the future work.

(II) Hypothesis testing. For the hypothesis testing problem

$$
H_{0}: \theta=\theta_{0} \quad \text { versus } \quad H_{1}: \theta=\theta_{1},
$$

where $\theta_{0}>0, \theta_{1}>0$. We can propose the test statistic

$$
\tilde{\Delta}_{n}=\frac{\Delta e^{\hat{\theta}_{n, \Delta}(n-1) \Delta}}{1-e^{-2 \hat{\theta}_{n, \Delta} \Delta}}\left(\hat{\theta}_{n, \Delta}-\theta_{0}\right) .
$$

Given $0<\alpha<1$, by Theorem 1.3, under $H_{0}$, for each $\rho>0$,

$$
\lim _{\Delta \rightarrow 0, n \rightarrow \infty} \sup _{x \in\left[0, \rho \beta_{n, \Delta}\right]}\left|\frac{P\left(\left|\tilde{\Delta}_{n}\right| \geq x\right)}{2-2 F_{\mathbb{C}}(x)}-1\right|=0 .
$$

Then, take the rejection region as $\left\{\left|\tilde{\Delta}_{n}\right| \geq t_{\alpha / 2}(1)\right\}$ and the corresponding probability $\tilde{\gamma}_{n, \Delta}$ of type II error can be written formulated as

$$
\tilde{\gamma}_{n, \Delta}=P\left(\left|\tilde{\Delta}_{n}\right|<t_{\alpha / 2}(1) \mid H_{1}\right):=P_{H_{1}}\left(\left|\tilde{\Delta}_{n}\right|<t_{\alpha / 2}(1)\right) .
$$

Applying Corollary 1.2, we can obtain the exponential decay rate for $\tilde{\gamma}_{n, \Delta}$.

Theorem 2.1. Let $b_{n, \Delta}$ be defined by (1.13). The type II error $\tilde{\gamma}_{n, \Delta}$ tends to 0 with exponential decay rate $e^{-r b_{n, \Delta}}$ for all $r>0$, i.e. $\lim _{\Delta \rightarrow 0, n \rightarrow \infty} \frac{\log \tilde{\gamma}_{n, \Delta}}{b_{n, \Delta}}=$ $-\infty$. 
Proof. We have

$$
\begin{aligned}
& \tilde{\gamma}_{n, \Delta} \\
& \leq P_{H_{1}}\left(\left|\frac{\Delta e^{\hat{\theta}_{n, \Delta}(n-1) \Delta}}{1-e^{-2 \hat{\theta}_{n, \Delta} \Delta}}\left(\hat{\theta}_{n, \Delta}-\theta_{1}\right)\right|^{\frac{1}{b_{n, \Delta}}}\right. \\
& \left.\geq\left(\frac{\Delta e^{\hat{\theta}_{n, \Delta}(n-1) \Delta}}{\left|1-e^{-2 \hat{\theta}_{n, \Delta} \Delta}\right|}\left|\theta_{1}-\theta_{0}\right|-t_{\alpha / 2}(1)\right)^{\frac{1}{b_{n, \Delta}}}\right) \\
& \leq P\left(\left|\hat{\theta}_{n, \Delta}-\theta_{1}\right| \geq \theta_{1} / 2 \mid H_{1}\right) \\
& +P_{H_{1}}\left(\left|\frac{\Delta e^{\hat{\theta}_{n, \Delta}(n-1) \Delta}}{1-e^{-2 \hat{\theta}_{n, \Delta} \Delta}}\left(\hat{\theta}_{n, \Delta}-\theta_{1}\right)\right|^{\frac{1}{b_{n, \Delta}}}\right. \\
& \left.\geq\left(\frac{\Delta e^{\theta_{1}(n-1) \Delta / 2}}{1-e^{-\theta_{1} \Delta}}\left|\theta_{1}-\theta_{0}\right|-t_{\alpha / 2}(1)\right)^{\frac{1}{b_{n, \Delta}}}\right) \text {. }
\end{aligned}
$$

Note that $\left(\frac{\Delta e^{\theta_{1}(n-1) \Delta / 2}}{1-e^{-\theta_{1} \Delta}}\left|\theta_{1}-\theta_{0}\right|-t_{\alpha / 2}(1)\right)^{\frac{1}{b_{n, \Delta}}}=O\left(e^{\frac{\theta_{1}(n-1) \Delta}{2 b_{n, \Delta}}}\right)$. Applying Theorem 1.2, we complete the proof of this theorem.

\subsection{Ergodic case: $\theta<0$}

(I) Confidence interval. An application of (1.19) gives the following approximate $(1-\alpha)(0<\alpha<1)$ confidence interval for $\theta$ :

$$
\left(\hat{\theta}_{n, \Delta}-\sqrt{\frac{-2 \hat{\theta}_{n, \Delta}}{n \Delta}} z_{\alpha / 2}, \hat{\theta}_{n, \Delta}+\sqrt{\frac{-2 \hat{\theta}_{n, \Delta}}{n \Delta}} z_{\alpha / 2}\right)
$$

where $z_{\alpha / 2}$ is the upper $\alpha / 2$-quantile of standard normal distribution. Here, the quality of approximation for above confidence interval can be an assessed by the Berry-Esseen bound of estimator $\hat{\theta}_{n, \Delta}([1],[11],[14],[15])$.

(II) Hypothesis testing. For the hypothesis testing problem

$$
H_{0}: \theta=\theta_{0} \quad \text { versus } \quad H_{1}: \theta=\theta_{1},
$$

for any $\theta_{0}<0, \theta_{1}<0$. We introduce the test statistic

$$
\hat{\Delta}_{n}=\sqrt{\frac{n \Delta}{-2 \hat{\theta}_{n, \Delta}}}\left(\hat{\theta}_{n, \Delta}-\theta_{0}\right) .
$$

Given $0<\alpha<1$, by Theorem 1.6, under $H_{0}$, for each $\rho>0$,

$$
\lim _{\Delta \rightarrow 0, n \rightarrow \infty} \sup _{x \in\left[0, \rho \lambda_{n, \Delta}\right]}\left|\frac{P\left(\left|\hat{\Delta}_{n}\right| \geq x\right)}{2-2 \Phi(x)}-1\right|=0 .
$$


Then, take the rejection region as $\left\{\left|\hat{\Delta}_{n}\right| \geq z_{\alpha / 2}\right\}$, and the corresponding probability $\hat{\gamma}_{n}$ of type II error can be written formulated as

$$
\hat{\gamma}_{n}=P_{H_{1}}\left(\left|\hat{\Delta}_{n}\right|<z_{\alpha / 2}\right) .
$$

Now, we can analyze its convergence rate explicitly.

Theorem 2.2. Let $\lambda_{n, \Delta}$ be defined by (1.16). The type II error $\hat{\gamma}_{n}$ tends to 0 with exponential decay rate $e^{-r \lambda_{n, \Delta}^{2}}$ for all $r>0$, i.e. $\lim _{\Delta \rightarrow 0, n \rightarrow \infty} \frac{\log \hat{\gamma}_{n}}{\lambda_{n, \Delta}^{2}}=-\infty$.

Proof. It holds that

$$
\begin{aligned}
\hat{\gamma}_{n} \leq & P_{H_{1}}\left(\left|\frac{1}{\lambda_{n, \Delta}} \sqrt{\frac{n \Delta}{-2 \hat{\theta}_{n, \Delta}}}\left(\hat{\theta}_{n, \Delta}-\theta_{1}\right)\right| \geq \frac{1}{\lambda_{n, \Delta}} \sqrt{\frac{n \Delta}{-2 \hat{\theta}_{n, \Delta}}}\left|\theta_{1}-\theta_{0}\right|-\frac{z_{\alpha / 2}}{\lambda_{n, \Delta}}\right) \\
\leq & P\left(\left|\hat{\theta}_{n, \Delta}-\theta_{1}\right| \geq \theta_{1} / 2 \mid H_{1}\right) \\
& +P_{H_{1}}\left(\left|\frac{1}{\lambda_{n, \Delta}} \sqrt{\frac{n \Delta}{-2 \hat{\theta}_{n, \Delta}}}\left(\hat{\theta}_{n, \Delta}-\theta_{1}\right)\right| \geq \frac{1}{\lambda_{n, \Delta}} \sqrt{\frac{n \Delta}{-3 \theta}}\left|\theta_{1}-\theta_{0}\right|-\frac{z_{\alpha / 2}}{\lambda_{n, \Delta}}\right) .
\end{aligned}
$$

By Corollary 1.3, we have $\lim _{\Delta \rightarrow 0, n \rightarrow \infty} \log \hat{\gamma}_{n} / \lambda_{n, \Delta}^{2}=-\infty$.

\subsection{Simulation study}

The sample path of (1.1) can be obtained easily by using the transition probability from $X_{t_{k}}$ to $X_{t_{k+1}}$, that is $X_{t_{k+1}}=e^{\theta \Delta} X_{t_{k}}+\epsilon_{k+1}^{\Delta}(\theta)$, where $t_{i}=i \Delta$, $\epsilon_{i}^{\Delta}(s):=e^{t_{i} \theta} \int_{t_{i-1}}^{t_{i}} e^{-\theta s} d W_{s} \sim N\left(0, \frac{1}{2 \theta}\left(e^{2 \Delta \theta}-1\right)\right),(i=0,1 \cdots, n-1)$. Here, we always simulate $N=1000$ times.

For the hypothesis testing (2.1) and (2.3), we conduct numerical simulations to evaluate the finite-sample performances of the following proposed tests

Proposed I: reject $H_{0}: \theta=\theta_{0}$, if $\left|\hat{\theta}_{n, \Delta}-\theta_{0}\right| \geq \frac{1-e^{-2 \hat{\theta}_{n, \Delta} \Delta}}{\Delta e^{\hat{\theta}_{n, \Delta}(n-1) \Delta}} t_{\alpha / 2}(1), \theta_{0}, \theta_{1}>0$

Proposed II: reject $H_{0}: \theta=\theta_{0}$, if $\left|\hat{\theta}_{n, \Delta}-\theta_{0}\right| \geq \sqrt{\frac{-2 \hat{\theta}_{n, \Delta}}{n \Delta}} z_{\alpha / 2}, \theta_{0}, \theta_{1}<0$

The size and power for the test statistics are summarized as follows. Tables 1-3 are for the explosive case, while the remainders (Tables 4-6) are for the stationary case. We can learn that

(i) In the explosive case, from Tables $1-3$, as $n \Delta$ or $\left|\theta_{0}-\theta_{1}\right|$ becomes larger (but $n \Delta$ not larger than 5 ), the size of $\tilde{\Delta}_{n}$ is close to the significance level 0.050 and the power increases dramatically to 1 . The reason is that the convergence speed of $\tilde{\Delta}_{n}$ is $e^{\theta_{0}(n-1) \Delta}$ under $H_{0}$ or $e^{\theta_{1}(n-1) \Delta}$ under $H_{1}$ (Theorem 1.2), which is quite fast. Moreover, by Theorem 2.1, $\tilde{\gamma}_{n, \Delta}$ tends to 0 
TABLE 1

(Explosive case) The Empirical C.I. means the empirical $1-\alpha$ confidence interval with confidence level $\alpha=0.05$. Here, take $\Delta=0.01$, significance level $\alpha=0.05$ with various sample number $n$.

\begin{tabular}{l|c|c|c|c}
\hline \multicolumn{3}{c}{$H_{0}: \theta_{0}=2$} & \multicolumn{3}{c}{ versus $H_{1}: \theta_{1}=3$} \\
Samples & Estimated $\hat{\theta}_{T}$ & Empirical C.I. & Power & Size \\
$n=200$ & 1.739 & {$[0.397,3.080]$} & 0.978 & 0.007 \\
$n=300$ & 1.968 & {$[1.834,2.102]$} & 1.000 & 0.036 \\
$n=400$ & 1.992 & {$[1.975,2.009]$} & 1.000 & 0.050 \\
$n=500$ & 1.999 & {$[1.997,2.001]$} & 1.000 & 0.051 \\
\hline
\end{tabular}

TABLE 2

(Explosive case) Take $n=100$, confidence and significance level $\alpha=0.05$, with varies iteration step $\Delta$.

\begin{tabular}{c|c|c|c|c}
\hline \multicolumn{4}{c}{$H_{0}: \theta_{0}=2$} & versus $H_{1}: \theta_{1}=3$ \\
Samples & Estimated $\hat{\theta}_{T}$ & Empirical C.I. & Power & Size \\
$\Delta=0.02$ & 1.710 & {$[0.338,3.083]$} & 0.983 & 0.007 \\
$\Delta=0.03$ & 1.939 & {$[1.800,2.077]$} & 1.000 & 0.044 \\
$\Delta=0.05$ & 1.999 & {$[1.998,2.002]$} & 1.000 & 0.053 \\
\hline
\end{tabular}

TABLE 3

(Explosive case) Take $\Delta=0.01, n=350$, significance level $\alpha=0.05$ with varies alternatives $H_{1}$.

\begin{tabular}{|c|c|c|}
\hline \multicolumn{2}{|c|}{$H_{0}: \theta_{0}=2$} & $H_{1}: \theta_{1}=\theta$ \\
\hline Samples & Power & Size \\
\hline$\theta=2.1$ & 0.985 & 0.055 \\
\hline$\theta=2.5$ & 1.000 & 0.055 \\
\hline$\theta=3.0$ & 1.000 & 0.055 \\
\hline
\end{tabular}

TABLE 4

(Stationary case) Take $\Delta=0.01$, confidence and significance level $\alpha=0.05$ with various sample number $n$.

\begin{tabular}{c|c|c|c|c}
\hline \multicolumn{4}{c}{$H_{0}: \theta_{0}=-2$} & versus $H_{1}: \theta_{1}=-3$ \\
Samples & Estimated $\hat{\theta}_{T}$ & Empirical C.I. & Power & Size \\
$n=5000$ & -2.043 & {$[-2.603,-1.482]$} & 0.881 & 0.064 \\
$n=6000$ & -2.040 & {$[-2.552,-1.529]$} & 0.939 & 0.061 \\
$n=7000$ & -2.026 & {$[-2.497,-1.554]$} & 0.965 & 0.050 \\
\hline
\end{tabular}

TABLE 5

(Stationary case) Take $n=2500$, confidence and significance level $\alpha=0.05$, with varies iteration step $\Delta$.

\begin{tabular}{c|c|c|c|c}
\hline \multicolumn{4}{c}{$H_{0}: \theta_{0}=-2$} & \multicolumn{3}{c}{$H_{1}: \theta_{1}=-3$} \\
Samples & Estimated $\hat{\theta}_{T}$ & Empirical C.I. & Power & Size \\
$\Delta=0.01$ & -2.081 & {$[-2.881,-1.282]$} & 0.578 & 0.049 \\
$\Delta=0.03$ & -2.027 & {$[-2.482,-1.571]$} & 0.971 & 0.052 \\
$\Delta=0.05$ & -2.016 & {$[-2.369,-1.664]$} & 0.996 & 0.055 \\
\hline
\end{tabular}

TABLE 6

(Stationary case) Take $\Delta=0.01, n=6500$, significance level $\alpha=0.05$ with varies alternatives $H_{1}$.

\begin{tabular}{c|c|l}
\hline$H_{0}: \theta_{0}=-2$ & versus & $H_{1}: \theta_{1}=\theta$ \\
Samples & Power & Size \\
$\theta=-2.1$ & 0.071 & 0.058 \\
$\theta=-2.5$ & 0.445 & 0.058 \\
$\theta=-3.0$ & 0.948 & 0.058 \\
$\theta=-4.0$ & 1.000 & 0.058 \\
\hline
\end{tabular}


with exponential decay rate $e^{-r b_{n, \Delta}}$ for all $r>0$, where $b_{n, \Delta}$ satisfies that

$$
b_{n, \Delta} \rightarrow \infty, \quad \frac{b_{n, \Delta}}{n \Delta} \rightarrow 0 .
$$

(ii) In the stationary case, from Tables $4-6$, as $n \Delta$ or $\left|\theta_{0}-\theta_{1}\right|$ becomes larger, the size of $\hat{\Delta}_{n}$ is close to the significance level 0.050 , and the power increases gradually to 1 which is slower than the explosive case. The above statement asserts our theory (Theorem 1.5, Theorem 2.2).

\section{Proofs of the main results}

For the Ornstein-Uhlenbeck process (1.1), the following formulas hold immediately

$$
X_{i}=e^{\theta t_{i}} \int_{0}^{t_{i}} e^{-\theta t} d W_{t}
$$

and

$$
X_{i}=e^{\theta \Delta} X_{i-1}+\epsilon_{i}^{\Delta}(\theta),
$$

where $\epsilon_{i}^{\Delta}(\theta):=e^{t_{i} \theta} \int_{t_{i-1}}^{t_{i}} e^{-\theta s} d W_{s} \sim N\left(0, \frac{1}{2 \theta}\left(e^{2 \Delta \theta}-1\right)\right), i=1,2, \cdots, n$.

\subsection{Explosive case: $\theta>0$}

In this subsection, we will prove Theorem 1.1, Theorem 1.2, Theorem 1.3, Corollary 1.1 and Corollary 1.2. To begin with, it is crucial to note that on the event $\frac{\sum_{i=1}^{n} X_{i} X_{i-1}}{\sum_{i=1}^{n} X_{i-1}^{2}}>0$

$$
\frac{\Delta e^{\theta(n-1) \Delta}}{1-e^{-2 \theta \Delta}}\left(\hat{\theta}_{n, \Delta}-\theta\right)=\frac{e^{\theta(n-1) \Delta}}{1-e^{-2 \theta \Delta}} \log \left(1+\Delta e^{-\theta n \Delta} \frac{e^{-\theta(n+1) \Delta} U_{n}}{\Delta e^{-2 \theta n \Delta} V_{n}}\right),
$$

where

$$
U_{n}=\sum_{i=1}^{n} \epsilon_{i}^{\Delta}(\theta) X_{i-1}, \quad V_{n}=\sum_{i=1}^{n} X_{i-1}^{2} .
$$

Propositions 3.1 plays an important role in our following analysis, and its proof will be given in Section 4 .

Proposition 3.1. Under assumption $(H)$, let $\beta_{n, \Delta}$ satisfy (1.5). Then, for each $\rho>0$, uniformly for $0 \leq x \leq \rho \beta_{n, \Delta}$,

$$
\frac{1}{1-F_{\mathbb{C}}(x)} P\left(\frac{e^{-\theta(n+2) \Delta} U_{n}}{\left(1-e^{-2 \theta \Delta}\right) e^{-2 \theta n \Delta} V_{n}} \geq x\right) \rightarrow 1
$$


and

$$
\frac{1}{F_{\mathbb{C}}(-x)} P\left(\frac{e^{-\theta(n+2) \Delta} U_{n}}{\left(1-e^{-2 \theta \Delta}\right) e^{-2 \theta n \Delta} V_{n}} \leq-x\right) \rightarrow 1
$$

\subsubsection{Proof of Theorem 1.1}

By using (1.2) and (3.3), we have

$$
\frac{\sum_{i=1}^{n} X_{i} X_{i-1}}{\sum_{i=1}^{n} X_{i-1}^{2}}=e^{\theta \Delta}\left(1+\Delta e^{-\theta n \Delta} \frac{e^{-\theta(n+1) \Delta} U_{n}}{\Delta e^{-2 \theta n \Delta} V_{n}}\right) .
$$

Then, it follows that

$$
\begin{aligned}
P\left(\frac{\sum_{i=1}^{n} X_{i} X_{i-1}}{\sum_{i=1}^{n} X_{i-1}^{2}} \leq 0\right) & =P\left(e^{\theta \Delta}\left(1+\Delta e^{-\theta n \Delta} \frac{e^{-\theta(n+1) \Delta} U_{n}}{\Delta e^{-2 \theta n \Delta} V_{n}}\right) \leq 0\right) \\
& \leq P\left(\left|\Delta e^{-\theta n \Delta} \frac{e^{-\theta(n+1) \Delta} U_{n}}{\Delta e^{-2 \theta n \Delta} V_{n}}\right| \geq 1\right) \\
& \leq P\left(\left|\frac{e^{-\theta(n+2) \Delta} U_{n}}{\left(1-e^{-2 \theta \Delta}\right) e^{-2 \theta n \Delta} V_{n}}\right| \geq \frac{e^{\theta(n-1) \Delta}}{\left(1-e^{-2 \theta \Delta}\right)}\right) .
\end{aligned}
$$

Together with (1.5) and (3.8), we can get

$$
\lim _{\Delta \rightarrow 0, n \rightarrow \infty} \beta_{n, \Delta} P\left(\frac{\sum_{i=1}^{n} X_{i} X_{i-1}}{\sum_{i=1}^{n} X_{i-1}^{2}} \leq 0\right)
$$

which completes the proof of this theorem.

\subsubsection{Equivalence in Cramér-type moderate deviations}

As an application of Theorem 1.1 and Proposition 3.1, we can get the following key result.

Lemma 3.1. For $\beta_{n, \Delta}$ defined by (1.5), we have

$$
\begin{gathered}
\lim _{\Delta \rightarrow 0, n \rightarrow \infty} \beta_{n, \Delta} P\left(\left|\frac{\Delta e^{\theta(n-1) \Delta}}{1-e^{-2 \theta \Delta}}\left(\hat{\theta}_{n, \Delta}-\theta\right)-\frac{e^{-\theta(n+2) \Delta} U_{n}}{\left(1-e^{-2 \theta \Delta}\right) e^{-2 \theta n \Delta} V_{n}}\right|\right. \\
\left.\geq \frac{1}{\beta_{n, \Delta} \log \beta_{n, \Delta}}\right)=0
\end{gathered}
$$

Proof. Using (3.3) and the fact $|\log (1+x)-x| \leq x^{2}, x>-\frac{1}{2}$, we can obtain for any $\epsilon>0$

$$
P\left(\left|\frac{\Delta e^{\theta(n-1) \Delta}}{1-e^{-2 \theta \Delta}}\left(\hat{\theta}_{n, \Delta}-\theta\right)-\frac{e^{-\theta(n+2) \Delta} U_{n}}{\left(1-e^{-2 \theta \Delta}\right) e^{-2 \theta n \Delta} V_{n}}\right| \geq \frac{1}{\beta_{n, \Delta} \log \beta_{n, \Delta}}\right)
$$




$$
\begin{aligned}
\leq & P\left(\frac{\sum_{i=1}^{n} X_{i} X_{i-1}}{\sum_{i=1}^{n} X_{i-1}^{2}} \leq 0\right)+P\left(\Delta e^{-\theta(n-1) \Delta} \frac{e^{-\theta(n+1) \Delta} U_{n}}{\Delta e^{-2 \theta n \Delta} V_{n}} \leq-\frac{1}{2}\right) \\
& +P\left(\frac{e^{\theta(n-1) \Delta}}{1-e^{-2 \theta \Delta}}\left(\Delta e^{-\theta n \Delta} \frac{e^{-\theta(n+1) \Delta} U_{n}}{\Delta e^{-2 \theta n \Delta} V_{n}}\right)^{2} \geq \frac{1}{\beta_{n, \Delta} \log \beta_{n, \Delta}}\right) \\
\leq & P\left(\frac{\sum_{i=1}^{n} X_{i} X_{i-1}}{\sum_{i=1}^{n} X_{i-1}^{2}} \leq 0\right)+P\left(\left|\frac{e^{-\theta(n+2) \Delta} U_{n}}{\left(1-e^{-2 \theta \Delta}\right) e^{-2 \theta n \Delta} V_{n}}\right| \geq \frac{e^{\theta n \Delta}}{2\left(1-e^{-2 \theta \Delta}\right)}\right) \\
& +P\left(\left(\frac{e^{-\theta(n+2) \Delta} U_{n}}{\left(1-e^{-2 \theta \Delta}\right) e^{-2 \theta n \Delta} V_{n}}\right)^{2} \geq \frac{e^{\theta(n-2) \Delta}}{\left(1-e^{-2 \theta \Delta}\right) \beta_{n, \Delta} \log \beta_{n, \Delta}}\right) \\
\leq & P\left(\frac{\sum_{i=1}^{n} X_{i} X_{i-1}}{\sum_{i=1}^{n} X_{i-1}^{2}} \leq 0\right)+2 P\left(\left|\frac{e^{-\theta(n+2) \Delta} U_{n}}{\left(1-e^{-2 \theta \Delta}\right) \Delta e^{-2 \theta n \Delta} V_{n}}\right| \geq \frac{\beta_{n, \Delta}}{\epsilon}\right) .
\end{aligned}
$$

Applying Theorem 1.1, Proposition 3.1 and the fact that

$$
\lim _{x \rightarrow+\infty} x\left(1-F_{\mathbb{C}}(x)\right)=\frac{1}{\pi},
$$

we can complete the proof of (3.7).

\subsubsection{Proof of Theorem 1.2}

By (3.8), for any $\epsilon>0$, there exists some positive constant $M$ such that

$$
\sup _{x \geq M}\left|\pi x\left(1-F_{\mathbb{C}}(x)\right)-1\right| \leq \epsilon .
$$

Now, for each $\rho>0$ and $2 M \leq x \leq \rho \beta_{n, \Delta}$, we have

$$
\begin{aligned}
& P\left(\frac{e^{-\theta(n+2) \Delta} U_{n}}{\left(1-e^{-2 \theta \Delta}\right) e^{-2 \theta n \Delta} V_{n}} \geq x+\frac{2}{\beta_{n, \Delta} \log \beta_{n, \Delta}}\right) \\
& \quad-P\left(\left|\frac{\Delta e^{\theta(n-1) \Delta}}{1-e^{-2 \theta \Delta}}\left(\hat{\theta}_{n, \Delta}-\theta\right)-\frac{e^{-\theta(n+2) \Delta} U_{n}}{\left(1-e^{-2 \theta \Delta}\right) e^{-2 \theta n \Delta} V_{n}}\right| \geq \frac{2}{\beta_{n, \Delta} \log \beta_{n, \Delta}}\right) \\
& \leq P\left(\frac{\Delta e^{\theta(n-1) \Delta}}{1-e^{-2 \theta \Delta}}\left(\hat{\theta}_{n, \Delta}-\theta\right) \geq x\right) \\
& \leq P\left(\frac{e^{-\theta(n+2) \Delta} U_{n}}{\left(1-e^{-2 \theta \Delta}\right) e^{-2 \theta n \Delta} V_{n}} \geq x-\frac{2}{\beta_{n, \Delta} \log \beta_{n, \Delta}}\right) \\
& \quad+P\left(\left|\frac{\Delta e^{\theta(n-1) \Delta}}{1-e^{-2 \theta \Delta}}\left(\hat{\theta}_{n, \Delta}-\theta\right)-\frac{e^{-\theta(n+2) \Delta} U_{n}}{\left(1-e^{-2 \theta \Delta}\right) e^{-2 \theta n \Delta} V_{n}}\right| \geq \frac{2}{\beta_{n, \Delta} \log \beta_{n, \Delta}}\right) .
\end{aligned}
$$


Firstly, by using Lemma 3.1 and (4.24), we have

$$
\begin{aligned}
& \lim _{\Delta \rightarrow 0, n \rightarrow \infty} \sup _{M \leq x \leq \rho \beta_{n, \Delta}} \frac{P\left(\left|\frac{\Delta e^{\theta(n-1) \Delta}}{1-e^{-2 \theta \Delta}}\left(\hat{\theta}_{n, \Delta}-\theta\right)-\frac{e^{-\theta(n+2) \Delta} U_{n}}{\left(1-e^{-2 \theta \Delta}\right) e^{-2 \theta n \Delta} V_{n}}\right| \geq \frac{2}{\beta_{n, \Delta} \log \beta_{n, \Delta}}\right)}{1-F_{\mathbb{C}}(x)} \\
& =0 .
\end{aligned}
$$

Secondly, from then mean value theorem and (4.24), it follows that,

$$
\sup _{2 M \leq x \leq \rho \beta_{n, \Delta}}\left|\frac{1-F_{\mathbb{C}}\left(x \pm \frac{2}{\beta_{n, \Delta} \log \beta_{n, \Delta}}\right)}{1-F_{\mathbb{C}}(x)}-1\right| \leq \frac{2 \pi \rho}{\left(1+2 M^{2}\right)(1-\epsilon) \log \beta_{n, \Delta}} .
$$

Then, we get

$$
\begin{aligned}
\sup _{2 M \leq x \leq \rho \beta_{n, \Delta}}\left|\frac{P\left(\frac{e^{-\theta(n+2) \Delta} U_{n}}{\left(1-e^{-2 \theta \Delta}\right) e^{-2 \theta} V_{n}} \geq x \pm \frac{2}{\beta_{n, \Delta} \log \beta_{n, \Delta}}\right)}{1-F_{\mathbb{C}}(x)}-1\right| \\
\leq \sup _{2 M \leq x \leq \rho \beta_{n, \Delta}}\left|\frac{1-F_{\mathbb{C}}\left(x \pm \frac{2}{\beta_{n, \Delta} \log \beta_{n, \Delta}}\right)}{1-F_{\mathbb{C}}(x)}-1\right| \\
+\sup _{2 M \leq x \leq \rho \beta_{n, \Delta}}\left|\frac{P\left(\frac{e^{-\theta(n+2) \Delta} U_{n}}{\left(1-e^{-2 \theta \Delta}\right) e^{-2 \theta n} V_{n}} \geq x \pm \frac{2}{\beta_{n, \Delta} \log \beta_{n, \Delta}}\right)}{1-F_{\mathbb{C}}\left(x \pm \frac{2}{\beta_{n, \Delta} \log \beta_{n, \Delta}}\right)}-1\right| \\
\cdot\left|\frac{1-F_{\mathbb{C}}\left(x \pm \frac{2}{\beta_{n, \Delta} \log \beta_{n, \Delta}}\right)}{1-F_{\mathbb{C}}(x)}\right| .
\end{aligned}
$$

Together with Proposition 3.1 and (3.12), we can obtain that

$$
\lim _{\Delta \rightarrow 0, n \rightarrow \infty} \sup _{2 M \leq x \leq \rho \beta_{n, \Delta}}\left|\frac{P\left(\frac{e^{-\theta(n+2) \Delta} U_{n}}{\left(1-e^{-2 \theta \Delta}\right) e^{-2 \theta n \Delta} V_{n}} \geq x \pm \frac{2}{\beta_{n, \Delta} \log \beta_{n, \Delta}}\right)}{1-F_{\mathbb{C}}(x)}-1\right|=0 .
$$

Therefore, by (3.10)-(3.13), it holds that

$$
\lim _{\Delta \rightarrow 0, n \rightarrow \infty} \sup _{2 M \leq x \leq \rho \beta_{n, \Delta}}\left|\frac{1}{1-F_{\mathbb{C}}(x)} P\left(\frac{\Delta e^{\theta(n-1) \Delta}}{1-e^{-2 \theta \Delta}}\left(\hat{\theta}_{n, \Delta}-\theta\right) \geq x\right)-1\right|=0 .
$$

Moreover, since the function $1-F_{\mathbb{C}}(x)$ is uniformly continuous, the standard discretized approximation argument shows that

$$
\lim _{\Delta \rightarrow 0, n \rightarrow \infty} \sup _{x \in \mathbb{R}}\left|P\left(\frac{\Delta e^{\theta(n-1) \Delta}}{1-e^{-2 \theta \Delta}}\left(\hat{\theta}_{n, \Delta}-\theta\right) \geq x\right)-\left(1-F_{\mathbb{C}}(x)\right)\right|=0 .
$$


Therefore, it follows that

$$
\lim _{\Delta \rightarrow 0, n \rightarrow \infty} \sup _{0 \leq x \leq 2 M}\left|\frac{1}{1-F_{\mathbb{C}}(x)} P\left(\frac{\Delta e^{\theta(n-1) \Delta}}{1-e^{-2 \theta \Delta}}\left(\hat{\theta}_{n, \Delta}-\theta\right) \geq x\right)-1\right|=0 .
$$

Finally, (1.6) can be obtained immediately by (3.14) and (3.15). Using the same procedure, we can prove (1.7), and the details are omitted here.

\subsubsection{Proof of Corollary 1.1}

In the sequel, we will show (1.8), while the proof of (1.9) is similar and the details are omitted here. In fact, we have

$$
\begin{aligned}
& \left|\frac{1}{1-F_{\mathbb{C}}(x)} P\left(\frac{e^{\theta n \Delta}}{2 \theta}\left(\hat{\theta}_{n, \Delta}-\theta\right) \geq x\right)-1\right| \\
& \leq\left|\frac{1-F_{\mathbb{C}}\left(\Upsilon_{n} x\right)}{1-F_{\mathbb{C}}(x)}\right| \cdot\left|\frac{1}{1-F_{\mathbb{C}}\left(\Upsilon_{n} x\right)} P\left(\frac{\Delta e^{\theta(n-1) \Delta}}{1-e^{-2 \theta \Delta}}\left(\hat{\theta}_{n, \Delta}-\theta\right) \geq \Upsilon_{n} x\right)-1\right| \\
& \quad+\left|\frac{1-F_{\mathbb{C}}\left(\Upsilon_{n} x\right)}{1-F_{\mathbb{C}}(x)}-1\right|,
\end{aligned}
$$

where $\Upsilon_{n}=\frac{2 \theta \Delta}{e^{\theta \Delta}-e^{-\theta \Delta}}=1+O\left(\Delta^{2}\right)$.

By Theorem 1.2, we only need to show

$$
\lim _{\Delta \rightarrow 0, n \rightarrow \infty} \sup _{0 \leq x \leq \rho \beta_{n, \Delta}}\left|\frac{1-F_{\mathbb{C}}\left(\Upsilon_{n} x\right)}{1-F_{\mathbb{C}}(x)}-1\right|=0 .
$$

For positive constant $M$ satisfying (4.24), we have

$$
\sup _{2 M \leq x \leq \rho \beta_{n, \Delta}}\left|\frac{1-F_{\mathbb{C}}\left(\Upsilon_{n} x\right)}{1-F_{\mathbb{C}}(x)}-1\right| \leq \frac{\rho^{2} \beta_{n, \Delta}^{2}\left|1-\Upsilon_{n}\right|}{1+2 M^{2}} \leq \frac{C \beta_{n, \Delta}^{2} \Delta^{2}}{1+2 M^{2}},
$$

which implies that

$$
\lim _{\Delta \rightarrow 0, n \rightarrow \infty} \sup _{2 M \leq x \leq \rho \beta_{n, \Delta}}\left|\frac{1-F_{\mathbb{C}}\left(\Upsilon_{n} x\right)}{1-F_{\mathbb{C}}(x)}-1\right|=0 .
$$

Finally, it is easy to get that

$$
\lim _{\Delta \rightarrow 0, n \rightarrow \infty} \sup _{0 \leq x \leq 2 M}\left|\frac{1-F_{\mathbb{C}}\left(\Upsilon_{n} x\right)}{1-F_{\mathbb{C}}(x)}-1\right|=0 .
$$

Consequently, we can complete the proof of this corollary. 


\subsubsection{Proof of Theorem 1.3}

To begin with, we can get the following formula

$$
\begin{aligned}
& \frac{\Delta e^{\hat{\theta}_{n, \Delta}(n-1) \Delta}}{1-e^{-2 \hat{\theta}_{n, \Delta} \Delta}}\left(\hat{\theta}_{n, \Delta}-\theta\right) \\
& =\frac{\Delta e^{\theta(n-1) \Delta}}{1-e^{-2 \theta \Delta}}\left(\hat{\theta}_{n, \Delta}-\theta\right)+\frac{\Delta e^{\theta(n-1) \Delta}}{1-e^{-2 \theta \Delta}}\left(\hat{\theta}_{n, \Delta}-\theta\right) \frac{e^{-2 \hat{\theta}_{n, \Delta} \Delta}-e^{-2 \theta \Delta}}{1-e^{-2 \hat{\theta}_{n, \Delta} \Delta}} \\
& \quad+\frac{\Delta e^{\theta(n-1) \Delta}}{1-e^{-2 \theta \Delta}}\left(\hat{\theta}_{n, \Delta}-\theta\right)\left(e^{\left(\hat{\theta}_{n, \Delta}-\theta\right)(n-1) \Delta}-1\right) \frac{1-e^{-2 \theta \Delta}}{1-e^{-2 \hat{\theta}_{n, \Delta} \Delta}} .
\end{aligned}
$$

By Theorem 1.2 and the procedures in its proof, to get (1.11), it is sufficient to show

$$
\begin{aligned}
& \lim _{\Delta \rightarrow 0, n \rightarrow \infty} \beta_{n, \Delta} P\left(\left|\frac{\Delta e^{\theta(n-1) \Delta}}{1-e^{-2 \theta \Delta}}\left(\hat{\theta}_{n, \Delta}-\theta\right)\right|\left|\frac{1-e^{-2 \theta \Delta}}{1-e^{-2 \hat{\theta}_{n, \Delta} \Delta}}-1\right| \geq \frac{1}{\beta_{n, \Delta} \log \beta_{n, \Delta}}\right) \\
& =0
\end{aligned}
$$

and

$$
\begin{gathered}
\lim _{\Delta \rightarrow 0, n \rightarrow \infty} \beta_{n, \Delta} P\left(\left|\frac{\Delta e^{\theta(n-1) \Delta}}{1-e^{-2 \theta \Delta}}\left(\hat{\theta}_{n, \Delta}-\theta\right)\right|\left|\left(e^{\left(\hat{\theta}_{n, \Delta}-\theta\right)(n-1) \Delta}-1\right) \frac{1-e^{-2 \theta \Delta}}{1-e^{-2 \hat{\theta}_{n, \Delta} \Delta}}\right|\right. \\
\left.\geq \frac{1}{\beta_{n, \Delta} \log \beta_{n, \Delta}}\right)=0 .
\end{gathered}
$$

In fact, for any $L>0$, we have

$$
\begin{aligned}
& P\left(\left|\frac{\Delta e^{\theta(n-1) \Delta}}{1-e^{-2 \theta \Delta}}\left(\hat{\theta}_{n, \Delta}-\theta\right)\right|\left|\frac{1-e^{-2 \theta \Delta}}{1-e^{-2 \hat{\theta}_{n, \Delta} \Delta}}-1\right| \geq \frac{1}{\beta_{n, \Delta} \log \beta_{n, \Delta}}\right) \\
& \leq P\left(\left|\frac{\Delta e^{\theta(n-1) \Delta}}{1-e^{-2 \theta \Delta}}\left(\hat{\theta}_{n, \Delta}-\theta\right)\right| \geq L \beta_{n, \Delta}\right) \\
& \quad+P\left(\left|\frac{1-e^{-2 \theta \Delta}}{1-e^{-2 \hat{\theta}_{n, \Delta} \Delta}}-1\right| \geq \frac{1}{L \beta_{n, \Delta}^{2} \log \beta_{n, \Delta}}\right) .
\end{aligned}
$$

By using Theorem 1.2 and (3.8), we have

$$
\lim _{\Delta \rightarrow 0, n \rightarrow \infty} \beta_{n, \Delta} P\left(\left|\frac{\Delta e^{\theta(n-1) \Delta}}{1-e^{-2 \theta \Delta}}\left(\hat{\theta}_{n, \Delta}-\theta\right)\right| \geq L \beta_{n, \Delta}\right) \leq \frac{1}{\pi L},
$$

which implies

$$
\lim _{L \rightarrow \infty} \lim _{\Delta \rightarrow 0, n \rightarrow \infty} \beta_{n, \Delta} P\left(\left|\frac{\Delta e^{\theta(n-1) \Delta}}{1-e^{-2 \theta \Delta}}\left(\hat{\theta}_{n, \Delta}-\theta\right)\right| \geq L \beta_{n, \Delta}\right)=0 .
$$

Moreover,

$$
P\left(\left|\frac{1-e^{-2 \theta \Delta}}{1-e^{-2 \hat{\theta}_{n, \Delta} \Delta}}-1\right| \geq \frac{1}{L \beta_{n, \Delta}^{2} \log \beta_{n, \Delta}}\right)
$$




$$
\begin{aligned}
\leq & P\left(\left|\hat{\theta}_{n, \Delta}-\theta\right| \geq \theta / 2\right) \\
& +P\left(\frac{2 e^{-2 \theta \Delta}}{1-e^{-\theta \Delta}}\left(\Delta\left|\hat{\theta}_{n, \Delta}-\theta\right|+\Delta^{2}\left|\hat{\theta}_{n, \Delta}-\theta\right|^{2}\right) \geq \frac{1}{L \beta_{n, \Delta}^{2} \log \beta_{n, \Delta}}\right) .
\end{aligned}
$$

From Theorem 1.2 and (3.8), we have

$$
\lim _{\Delta \rightarrow 0, n \rightarrow \infty} \beta_{n, \Delta} P\left(\left|\frac{1-e^{-2 \theta \Delta}}{1-e^{-2 \hat{\theta}_{n, \Delta} \Delta}}-1\right| \geq \frac{1}{L \beta_{n, \Delta}^{2} \log \beta_{n, \Delta}}\right)=0 .
$$

Therefore, together with (3.19) and (3.20), we can prove (3.17).

Finally, (3.18) can be proved similarly, and the details are omitted here.

\subsubsection{Proof of Corollary 1.2}

Notice that for any $x \geq 0$,

$P\left(\left|\frac{e^{\theta n \Delta}}{2 \theta}\left(\hat{\theta}_{n, \Delta}-\theta\right)\right|^{\frac{1}{b_{n, \Delta}}} \geq x\right)=P\left(\left|\frac{\Delta e^{\theta(n-1) \Delta}}{1-e^{-2 \theta \Delta}}\left(\hat{\theta}_{n, \Delta}-\theta\right)\right| \geq\left|\Upsilon_{n}\right| x^{b_{n, \Delta}}\right)$,

where $\Upsilon_{n}=\frac{2 \theta \Delta}{e^{\theta \Delta}-e^{-\theta \Delta}}=1+O\left(\Delta^{2}\right)$.

In the case of $0 \leq x<1$, we have $x^{b_{n, \Delta}} \rightarrow 0$ as $n \rightarrow \infty$. Then, Theorem 1.2 implies that

$$
\frac{P\left(\left|\frac{e^{\theta n \Delta}}{2 \theta}\left(\hat{\theta}_{n, \Delta}-\theta\right)\right|^{\frac{1}{b_{n, \Delta}}} \geq x\right)}{1-F_{\mathbb{C}}\left(\left|\Upsilon_{n}\right| x^{b_{n, \Delta}}\right)} \rightarrow 1, \quad n \rightarrow \infty .
$$

Hence, we can obtain that

$$
\begin{aligned}
& \lim _{\Delta \rightarrow 0, n \rightarrow \infty} \frac{1}{b_{n, \Delta}} \log P\left(\left|\frac{e^{\theta n \Delta}}{2 \theta}\left(\hat{\theta}_{n, \Delta}-\theta\right)\right|^{\frac{1}{b_{n, \Delta}}} \geq x\right) \\
& =\lim _{\Delta \rightarrow 0, n \rightarrow \infty} \frac{1}{b_{n, \Delta}} \log \left(1-F_{\mathbb{C}}\left(\left|\Upsilon_{n}\right| x^{b_{n, \Delta}}\right)\right)=0 .
\end{aligned}
$$

In the case of $x=1$, we have $x^{b_{n, \Delta}}=1$. By Theorem 1.2, it follows that

$$
\frac{P\left(\left|\frac{e^{\theta n \Delta}}{2 \theta}\left(\hat{\theta}_{n, \Delta}-\theta\right)\right|^{\frac{1}{b_{n, \Delta}}} \geq x\right)}{1-F_{\mathbb{C}}\left(\left|\Upsilon_{n}\right|\right)} \rightarrow 1, \quad n \rightarrow \infty .
$$

Therefore,

$$
\begin{aligned}
& \lim _{\Delta \rightarrow 0, n \rightarrow \infty} \frac{1}{b_{n, \Delta}} \log P\left(\left|\frac{e^{\theta n \Delta}}{2 \theta}\left(\hat{\theta}_{n, \Delta}-\theta\right)\right|^{\frac{1}{b_{n, \Delta}}} \geq x\right) \\
& =\lim _{\Delta \rightarrow 0, n \rightarrow \infty} \frac{1}{b_{n, \Delta}} \log \left(1-F_{\mathbb{C}}\left(\left|\Upsilon_{n}\right|\right)\right)=0 .
\end{aligned}
$$


In the case of $x>1$, if $b_{n, \Delta}$ satisfies condition (1.13), it holds that

$$
x^{b_{n, \Delta}} \rightarrow \infty, \quad \frac{n \Delta x^{b_{n, \Delta}} b_{n, \Delta}^{4}}{e^{2 \theta n \Delta}} \rightarrow 0 .
$$

By Theorem 1.2, we have

$$
\frac{P\left(\left|\frac{e^{\theta n \Delta}}{2 \theta}\left(\hat{\theta}_{n, \Delta}-\theta\right)\right|^{\frac{1}{b_{n, \Delta}}} \geq x\right)}{1-F_{\mathbb{C}}\left(\left|\Upsilon_{n}\right| x^{b_{n, \Delta}}\right)} \rightarrow 1, \quad n \rightarrow \infty .
$$

According to (3.8),

$$
\begin{aligned}
& \lim _{\Delta \rightarrow 0, n \rightarrow \infty} \frac{1}{b_{n, \Delta}} \log P\left(\left|\frac{e^{\theta n \Delta}}{2 \theta}\left(\hat{\theta}_{n, \Delta}-\theta\right)\right|^{\frac{1}{b_{n, \Delta}}} \geq x\right) \\
& =\lim _{\Delta \rightarrow 0, n \rightarrow \infty} \frac{1}{b_{n, \Delta}} \log \left(1-F_{\mathbb{C}}\left(\left|\Upsilon_{n}\right| x^{b_{n, \Delta}}\right)\right)=-\log x .
\end{aligned}
$$

Then, the proof of (1.14) is completed by (3.21)-(3.23). Finally, by using Theorem 1.3 and the procedure in the proof of (1.14), we can show (1.15), and the details are omitted here.

\subsection{Ergodic case: $\theta<0$}

In this subsection, we will prove Theorem 1.4, Theorem 1.5, Theorem 1.6 and Corollary 1.3. The below formula plays an important in our following analysis.

$$
\sqrt{-\frac{n \Delta}{2 \theta}}\left(\hat{\theta}_{n, \Delta}-\theta\right)=\sqrt{-\frac{n \Delta}{2 \theta}} \cdot \frac{1}{\Delta} \log \left(1+\frac{\Delta}{\sqrt{n \Delta}} \frac{(n \Delta)^{-1 / 2} e^{-\theta \Delta} U_{n}}{n^{-1} V_{n}}\right)
$$

where $U_{n}, V_{n}$ are defined by (3.4).

The following results state that $\sqrt{-\frac{n \Delta}{2 \theta}}\left(\hat{\theta}_{n, \Delta}-\theta\right)$ is exponential equivalent to $\frac{(n \Delta)^{-1 / 2} e^{-\theta \Delta} U_{n}}{\sqrt{-2 \theta} n^{-1} V_{n}}$. The proof of moderate deviation for $\frac{(n \Delta)^{-1 / 2} e^{-\theta \Delta} U_{n}}{\sqrt{-2 \theta} n^{-1} V_{n}}$ (Proposition 3.2) will be postponed to Section 4 .

Proposition 3.2. Under assumption $\left(H^{\prime}\right)$, let $\lambda_{n, \Delta}$ satisfy (1.16). Then, for each $\rho>0$, uniformly for $0 \leq x \leq \rho \lambda_{n, \Delta}$,

$$
\frac{1}{1-\Phi(x)} P\left(\frac{(n \Delta)^{-1 / 2} e^{-\theta \Delta} U_{n}}{\sqrt{-2 \theta} n^{-1} V_{n}} \geq x\right) \rightarrow 1
$$

and

$$
\frac{1}{1-\Phi(x)} P\left(\frac{(n \Delta)^{-1 / 2} e^{-\theta \Delta} U_{n}}{\sqrt{-2 \theta} n^{-1} V_{n}} \leq-x\right) \rightarrow 1 .
$$




\subsubsection{Proof of Theorem 1.4}

Applying (1.2) and (3.24), we can obtain

$$
\frac{\sum_{i=1}^{n} X_{i} X_{i-1}}{\sum_{i=1}^{n} X_{i-1}^{2}}=e^{\theta \Delta}\left(1+\frac{\Delta}{\sqrt{n \Delta}} \frac{(n \Delta)^{-1 / 2} e^{-\theta \Delta} U_{n}}{n^{-1} V_{n}}\right) .
$$

Consequently, it holds that

$$
\begin{aligned}
P\left(\frac{\sum_{i=1}^{n} X_{i} X_{i-1}}{\sum_{i=1}^{n} X_{i-1}^{2}} \leq 0\right) & =P\left(e^{\theta \Delta}\left(1+\frac{\Delta}{\sqrt{n \Delta}} \frac{(n \Delta)^{-1 / 2} e^{-\theta \Delta} U_{n}}{n^{-1} V_{n}}\right) \leq 0\right) \\
& \leq P\left(\left|\frac{\Delta}{\sqrt{n \Delta}} \frac{(n \Delta)^{-1 / 2} e^{-\theta \Delta} U_{n}}{n^{-1} V_{n}}\right| \geq 1\right) \\
& \leq P\left(\left|\frac{(n \Delta)^{-1 / 2} e^{-\theta \Delta} U_{n}}{n^{-1} V_{n}}\right| \geq \sqrt{n / \Delta}\right) .
\end{aligned}
$$

From (1.16) and Proposition 3.2, we can completes the proof of this theorem.

\subsubsection{Exponential equivalence in Cramér-type moderate deviations}

Now, by Theorem 1.4 and Proposition 3.2, we can have the following exponential equivalence.

Lemma 3.2. Under assumption $\left(H^{\prime}\right)$, let $\lambda_{n, \Delta}$ satisfy (1.16), we have

$$
\begin{aligned}
& \lim _{\Delta \rightarrow 0, n \rightarrow \infty} \frac{1}{\lambda_{n, \Delta}^{2}} \log P\left(\left|\sqrt{-\frac{n \Delta}{2 \theta}}\left(\hat{\theta}_{n, \Delta}-\theta\right)-\frac{(n \Delta)^{-1 / 2} e^{-\theta \Delta} U_{n}}{\sqrt{-2 \theta} n^{-1} V_{n}}\right| \geq \frac{1}{\lambda_{n, \Delta} \log \lambda_{n, \Delta}}\right) \\
& =-\infty .
\end{aligned}
$$

Proof. Using (3.24) and the fact $|\log (1+x)-x| \leq x^{2}, x>-\frac{1}{2}$, we can obtain for any $L>0$

$$
\begin{aligned}
& P\left(\left|\sqrt{-\frac{n \Delta}{2 \theta}}\left(\hat{\theta}_{n, \Delta}-\theta\right)-\frac{(n \Delta)^{-1 / 2} e^{-\theta \Delta} U_{n}}{\sqrt{-2 \theta} n^{-1} V_{n}}\right| \geq \frac{1}{\lambda_{n, \Delta} \log \lambda_{n, \Delta}}\right) \\
& \leq P\left(\frac{\sum_{i=1}^{n} X_{i} X_{i-1}}{\sum_{i=1}^{n} X_{i-1}^{2}} \leq 0\right)+P\left(\frac{(n \Delta)^{-1 / 2} e^{-\theta \Delta} U_{n}}{n^{-1} V_{n}} \leq-\frac{1}{2} \sqrt{n / \Delta}\right) \\
& \quad+P\left(\left|\frac{(n \Delta)^{-1 / 2} e^{-\theta \Delta} U_{n}}{n^{-1} V_{n}}\right| \geq\left(\frac{\sqrt{n / \Delta}}{\lambda_{n, \Delta} \log \lambda_{n, \Delta}}\right)^{1 / 2}\right) \\
& \leq P\left(\frac{\sum_{i=1}^{n} X_{i} X_{i-1}}{\sum_{i=1}^{n} X_{i-1}^{2}} \leq 0\right)+2 P\left(\left|\frac{(n \Delta)^{-1 / 2} e^{-\theta \Delta} U_{n}}{n^{-1} V_{n}}\right| \geq L \lambda_{n, \Delta}\right) .
\end{aligned}
$$

From Theorem 1.4 and Proposition 3.2, it follows that

$$
\begin{aligned}
& \lim _{\Delta \rightarrow 0, n \rightarrow \infty} \frac{1}{\lambda_{n, \Delta}^{2}} \log P\left(\left|\sqrt{-\frac{n \Delta}{2 \theta}}\left(\hat{\theta}_{n, \Delta}-\theta\right)-\frac{(n \Delta)^{-1 / 2} e^{-\theta \Delta} U_{n}}{\sqrt{-2 \theta} n^{-1} V_{n}}\right| \geq \frac{1}{\lambda_{n, \Delta} \log \lambda_{n, \Delta}}\right) \\
& \leq-L^{2} / 2,
\end{aligned}
$$

which completes the proof of this lemma by letting $L \rightarrow \infty$. 


\subsubsection{Proof of Theorem 1.5}

Here, we only prove (1.20), since (1.21) can be obtained in the same way. Firstly, we can write that

$$
\begin{aligned}
& P\left(\sqrt{\frac{n \Delta}{-2 \theta}}\left(\hat{\theta}_{n, \Delta}-\theta\right) \geq x+\frac{1}{\lambda_{n, \Delta} \log \lambda_{n, \Delta}}\right) \\
& \quad-P\left(\left|\sqrt{-\frac{n \Delta}{2 \theta}}\left(\hat{\theta}_{n, \Delta}-\theta\right)-\frac{(n \Delta)^{-1 / 2} e^{-\theta \Delta} U_{n}}{\sqrt{-2 \theta} n^{-1} V_{n}}\right| \geq \frac{1}{\lambda_{n, \Delta} \log \lambda_{n, \Delta}}\right) \\
& \leq P\left(\sqrt{\frac{n \Delta}{-2 \theta}}\left(\hat{\theta}_{n, \Delta}-\theta\right) \geq x\right) \\
& \leq P\left(\sqrt{\frac{n \Delta}{-2 \theta}}\left(\hat{\theta}_{n, \Delta}-\theta\right) \geq x-\frac{1}{\lambda_{n, \Delta} \log \lambda_{n, \Delta}}\right) \\
& \quad+P\left(\left|\sqrt{-\frac{n \Delta}{2 \theta}}\left(\hat{\theta}_{n, \Delta}-\theta\right)-\frac{(n \Delta)^{-1 / 2} e^{-\theta \Delta} U_{n}}{\sqrt{-2 \theta} n^{-1} V_{n}}\right| \geq \frac{1}{\lambda_{n, \Delta} \log \lambda_{n, \Delta}}\right) .
\end{aligned}
$$

Note that for all $x \geq 0$,

$$
\frac{1}{2+\sqrt{2 \pi} x} e^{-x^{2} / 2} \leq 1-\Phi(x) \leq \frac{1}{2} e^{-x^{2} / 2} .
$$

Then, using Lemma 3.2, we have

$$
\begin{gathered}
\lim _{\Delta \rightarrow 0, n \rightarrow \infty} \sup _{0 \leq x \leq \rho \lambda_{n, \Delta}} \frac{1}{1-\Phi(x)} P\left(\left|\sqrt{-\frac{n \Delta}{2 \theta}}\left(\hat{\theta}_{n, \Delta}-\theta\right)-\frac{(n \Delta)^{-1 / 2} e^{-\theta \Delta} U_{n}}{\sqrt{-2 \theta} n^{-1} V_{n}}\right|\right. \\
\left.\geq \frac{1}{\lambda_{n, \Delta} \log \lambda_{n, \Delta}}\right)=0 .
\end{gathered}
$$

Moreover, letting $\Xi_{n}=\frac{1}{\lambda_{n, \Delta} \log \lambda_{n, \Delta}}$,

$$
\begin{aligned}
& \left|\frac{1}{1-\Phi(x)} P\left(\sqrt{\frac{n \Delta}{-2 \theta}}\left(\hat{\theta}_{n, \Delta}-\theta\right) \geq x \pm \Xi_{n}\right)\right| \\
& \leq\left|\frac{1-\Phi\left(x \pm \Xi_{n}\right)}{1-\Phi(x)}\right| \cdot\left|\frac{1}{1-\Phi\left(x \pm \Xi_{n}\right)} P\left(\sqrt{\frac{n \Delta}{-2 \theta}}\left(\hat{\theta}_{n, \Delta}-\theta\right) \geq x \pm \Xi_{n}\right)\right| \\
& \quad+\left|\frac{1-\Phi\left(x \pm \Xi_{n}\right)}{1-\Phi(x)}-1\right| .
\end{aligned}
$$

By (3.27) and mean value theorem, we have

$$
\sup _{0 \leq x \leq \rho \lambda_{n, \Delta}}\left|\frac{1-\Phi\left(x \pm \Xi_{n}\right)}{1-\Phi(x)}-1\right| \leq \frac{C}{\log \lambda_{n, \Delta}} .
$$


Together with Proposition 3.2,

$$
\lim _{\Delta \rightarrow 0, n \rightarrow \infty} \sup _{0 \leq x \leq \rho \lambda_{n, \Delta}}\left|\frac{1}{1-\Phi(x)} P\left(\sqrt{\frac{n \Delta}{-2 \theta}}\left(\hat{\theta}_{n, \Delta}-\theta\right) \geq x \pm \Xi_{n}\right)\right|=0 .
$$

Therefore, we can complete the proof of (1.20) by (3.28) and (3.29).

\subsubsection{Proof of Theorem 1.6}

By the method as in the proof of Theorem 1.5, we only need to show

$$
\begin{gathered}
\lim _{\Delta \rightarrow 0, n \rightarrow \infty} \frac{1}{\lambda_{n, \Delta}^{2}} \log P\left(\left|\sqrt{\frac{n \Delta}{-2 \hat{\theta}_{n, \Delta}}}\left(\hat{\theta}_{n, \Delta}-\theta\right)-\sqrt{\frac{n \Delta}{-2 \theta}}\left(\hat{\theta}_{n, \Delta}-\theta\right)\right|\right. \\
\left.\geq \frac{1}{\lambda_{n, \Delta} \log \lambda_{n, \Delta}}\right)=-\infty
\end{gathered}
$$

In fact, we have

$$
\sqrt{\frac{n \Delta}{-2 \hat{\theta}_{n, \Delta}}}\left(\hat{\theta}_{n, \Delta}-\theta\right)-\sqrt{\frac{n \Delta}{-2 \theta}}\left(\hat{\theta}_{n, \Delta}-\theta\right)=\frac{2\left(\hat{\theta}_{n, \Delta}-\theta\right)^{2}}{\sqrt{4 \hat{\theta}_{n, \Delta} \theta}\left(\sqrt{-2 \hat{\theta}_{n, \Delta}}+\sqrt{-2 \theta}\right)} .
$$

Hence, it holds that

$$
\begin{aligned}
& P\left(\left|\sqrt{\frac{n \Delta}{-2 \hat{\theta}_{n, \Delta}}}\left(\hat{\theta}_{n, \Delta}-\theta\right)-\sqrt{\frac{n \Delta}{-2 \theta}}\left(\hat{\theta}_{n, \Delta}-\theta\right)\right| \geq \frac{1}{\lambda_{n, \Delta} \log \lambda_{n, \Delta}}\right) \\
& \leq P\left(\left|\hat{\theta}_{n, \Delta}-\theta\right| \geq|\theta| / 2\right)+P\left(\frac{2 \sqrt{n \Delta}\left(\hat{\theta}_{n, \Delta}-\theta\right)^{2}}{2 \sqrt{-\theta^{3}}+\sqrt{2 \theta^{3}}} \geq \frac{1}{\lambda_{n, \Delta} \log \lambda_{n, \Delta}}\right) .
\end{aligned}
$$

Then, we can complete the proof by Theorem 1.5.

\subsubsection{Proof of Corollary 1.3}

For any $x>0$, take $y=\lambda_{n, \Delta} x$, and it holds by (3.27)

$$
\lim _{\Delta \rightarrow 0, n \rightarrow \infty} \frac{1}{\lambda_{n, \Delta}^{2}} \log (1-\Phi(y))=-\frac{x^{2}}{2} .
$$

Then, by virtue of Theorem 1.5,

$$
\lim _{\Delta \rightarrow 0, n \rightarrow \infty} \frac{1}{\lambda_{n, \Delta}^{2}} \log P\left(\frac{1}{\lambda_{n, \Delta}} \sqrt{\frac{n \Delta}{-2 \theta}}\left(\hat{\theta}_{n, \Delta}-\theta\right) \geq x\right)=-\frac{x^{2}}{2} .
$$

Similarly, we can also have by Theorem 1.6

$$
\lim _{\Delta \rightarrow 0, n \rightarrow \infty} \frac{1}{\lambda_{n, \Delta}^{2}} \log P\left(\frac{1}{\lambda_{n, \Delta}} \sqrt{\frac{n \Delta}{-2 \hat{\theta}_{n, \Delta}}}\left(\hat{\theta}_{n, \Delta}-\theta\right) \geq x\right)=-\frac{x^{2}}{2} .
$$




\section{Proofs of two technical propositions}

\subsection{Proof of Proposition 3.1}

Recall that

$$
U_{n}=\sum_{i=1}^{n} \epsilon_{i}^{\Delta}(\theta) X_{i-1}, \quad V_{n}=\sum_{i=1}^{n} X_{i-1}^{2} .
$$

The above two terms can be rewritten as

$$
e^{-\theta(n+2) \Delta} U_{n}=e^{-3 \theta \Delta} \widetilde{U}_{n} \widetilde{X}_{n}+r_{1 n},\left(1-e^{-2 \theta \Delta}\right) e^{-2 \theta n \Delta} V_{n}=e^{-2 \theta \Delta} \widetilde{X}_{n-1}^{2}-r_{2 n},
$$

where

$$
\begin{gathered}
\widetilde{U}_{n}=\sum_{i=1}^{n} e^{-\theta(n-i) \Delta} \epsilon_{i}^{\Delta}(\theta), \quad \widetilde{X}_{i}:=e^{-\theta i \Delta} X_{i}, \quad i=0, \cdots, n, \\
r_{1 n}=e^{-3 \theta \Delta} \sum_{i=1}^{n} e^{-\theta(n-i) \Delta} \epsilon_{i}^{\Delta}(\theta)\left(\widetilde{X}_{i-1}-\widetilde{X}_{n}\right)
\end{gathered}
$$

and

$$
r_{2 n}=e^{-2 \theta \Delta} \sum_{i=1}^{n} e^{-2 \theta(n-i+1) \Delta}\left(\widetilde{X}_{i-1}^{2}-\widetilde{X}_{i-2}^{2}\right) .
$$

Then, we have the following key decomposition for $\frac{e^{-\theta(n+2) \Delta} U_{n}}{\left(1-e^{-2 \theta \Delta}\right) e^{-2 \theta n \Delta} V_{n}}$.

Lemma 4.1. Define

$$
R_{1 n}=\frac{e^{-\theta \Delta} \widetilde{U}_{n}}{\widetilde{X}_{n-1}} \cdot \frac{e^{-\theta n \Delta} \epsilon_{n}^{\Delta}(\theta)}{\widetilde{X}_{n-1}}, \quad R_{2 n}=\frac{r_{1 n}}{e^{-2 \theta \Delta} \widetilde{X}_{n-1}^{2}-r_{2 n}},
$$

and

$$
R_{3 n}=\frac{r_{2 n}}{e^{-2 \theta \Delta} \widetilde{X}_{n-1}^{2}-r_{2 n}}
$$

We have

$$
\frac{e^{-\theta(n+2) \Delta} U_{n}}{\left(1-e^{-2 \theta \Delta}\right) e^{-2 \theta n \Delta} V_{n}}=\frac{e^{-n \Delta} \widetilde{U}_{n}}{\widetilde{X}_{n-1}}+R_{1 n}+R_{2 n}+\widetilde{R}_{3 n}
$$

where

$$
\widetilde{R}_{3 n}=\left(\frac{e^{-n \Delta} \widetilde{U}_{n}}{\widetilde{X}_{n-1}}+R_{1 n}\right) R_{3 n}
$$

Proof. Using (4.1), we have

$$
\frac{e^{-\theta(n+2) \Delta} U_{n}}{\left(1-e^{-2 \theta \Delta}\right) e^{-2 \theta n \Delta} V_{n}}-\frac{e^{-\theta \Delta} \widetilde{U}_{n} \widetilde{X}_{n}}{\widetilde{X}_{n-1}^{2}}=R_{2 n}+\frac{e^{-\theta \Delta} \widetilde{U}_{n} \widetilde{X}_{n}}{\widetilde{X}_{n-1}^{2}} R_{3 n} .
$$


Since $\widetilde{X}_{n}=\widetilde{X}_{n-1}+e^{-\theta n \Delta} \epsilon_{n}^{\Delta}(\theta)$, it holds that

$$
e^{-\theta \Delta}\left(\frac{\widetilde{U}_{n} \widetilde{X}_{n}}{\widetilde{X}_{n-1}^{2}}-\frac{\widetilde{U}_{n}}{\widetilde{X}_{n-1}}\right)=R_{1 n} .
$$

Then, (4.6) can be proved immediately.

Lemma 4.2. Under assumption $(H)$, let $\beta_{n, \Delta}$ satisfy (1.5). Then, for each $\rho>0$, uniformly for $0 \leq x \leq \rho \beta_{n, \Delta}$,

$$
\frac{1}{1-F_{\mathbb{C}}(x)} P\left(\frac{e^{-\theta \Delta} \widetilde{U}_{n}}{\widetilde{X}_{n-1}} \geq x\right) \rightarrow 1
$$

and

$$
\frac{1}{F_{\mathbb{C}}(-x)} P\left(\frac{e^{-\theta \Delta} \widetilde{U}_{n}}{\widetilde{X}_{n-1}} \leq-x\right) \rightarrow 1
$$

Proof. Note that $\left(e^{-\theta \Delta} \widetilde{U}_{n}, \widetilde{X}_{n-1}\right)$ is a two dimensional normal variable, satisfying

$$
\begin{gathered}
E \widetilde{X}_{n-1}=0, \quad \operatorname{Var}\left(\widetilde{X}_{n-1}\right)=\frac{1}{2 \theta}\left(1-e^{-2 \theta(n-1) \Delta}\right):=\sigma_{\widetilde{X}_{n-1}}^{2}, \\
E e^{-\theta \Delta} \widetilde{U}_{n}=0, \quad \operatorname{Var}\left(e^{-\theta \Delta} \widetilde{U}_{n}\right)=\frac{1}{2 \theta}\left(1-e^{-2 \theta n \Delta}\right):=\sigma_{e^{-\theta \Delta} \widetilde{U}_{n}}^{2},
\end{gathered}
$$

and

$$
\operatorname{Cov}\left(e^{-\theta \Delta} \widetilde{U}_{n}, \widetilde{X}_{n-1}\right)=\frac{1}{2 \theta}(n-1)\left(e^{2 \theta \Delta}-1\right) e^{-\theta(n+1) \Delta} .
$$

Letting

$$
\rho_{e^{-\theta \Delta} \widetilde{U}_{n}, \widetilde{X}_{n-1}}=\sigma_{\widetilde{X}_{n-1}}^{-1} \sigma_{e^{-\theta \Delta} \widetilde{U}_{n}}^{-1} \operatorname{Cov}\left(e^{-\theta \Delta} \widetilde{U}_{n}, \widetilde{X}_{n-1}\right),
$$

we can get that

$$
e^{-\theta \Delta} \widetilde{U}_{n}=\rho_{e^{-\theta \Delta} \widetilde{U}_{n}, \widetilde{X}_{n-1}} \sigma_{e^{-\theta \Delta} \widetilde{U}_{n}} \sigma_{\widetilde{X}_{n-1}}^{-1} \widetilde{X}_{n-1}+\sqrt{1-\rho_{e^{-\theta \Delta} \widetilde{U}_{n}, \widetilde{X}_{n-1}}^{2}} \sigma_{e^{-\theta \Delta} \widetilde{U}_{n}} \xi,
$$

where $\xi$ is a standard normal variable which is independent of $\widetilde{X}_{n-1}$. Therefore, it holds that

$$
\begin{aligned}
& \frac{e^{-\theta \Delta} \widetilde{U}_{n}}{\widetilde{X}_{n-1}} \\
& =\sqrt{1-\rho_{e^{-\theta \Delta} \widetilde{U}_{n}, \widetilde{X}_{n-1}}^{2}} \sigma_{e^{-\theta \Delta} \widetilde{U}_{n}} \sigma_{\widetilde{X}_{n-1}}^{-1} \frac{\xi}{\sigma_{\widetilde{X}_{n-1}}^{-1} \widetilde{X}_{n-1}}+\rho_{e^{-\theta \Delta} \widetilde{U}_{n}, \widetilde{X}_{n-1}} \sigma_{e^{-\theta \Delta} \widetilde{U}_{n}} \sigma_{\widetilde{X}_{n-1}}^{-1} \\
& =A_{n} \frac{\xi}{\sigma_{\widetilde{X}_{n-1}}^{-1} \widetilde{X}_{n-1}}+B_{n} .
\end{aligned}
$$


Moreover, we also have

$$
A_{n}=1+O\left((n \Delta)^{2} e^{-2 \theta n \Delta}\right), \quad B_{n}=O\left(n \Delta e^{-\theta n \Delta}\right) .
$$

Since $\frac{\xi}{\sigma_{\widetilde{X}_{n-1}}^{-1} \widetilde{X}_{n-1}} \sim \mathbb{C}$, we have $P\left(\frac{e^{-\theta \Delta} \widetilde{U}_{n}}{\tilde{X}_{n-1}} \geq x\right)=1-F_{\mathbb{C}}\left(A_{n}^{-1}\left(x-B_{n}\right)\right)$.

For any $\epsilon>0$, let $M$ be positive constant such that (4.24) holds. Then

$$
\begin{aligned}
& \sup _{2 M \leq x \leq \rho \beta_{n, \Delta}}\left|\frac{1}{1-F_{\mathbb{C}}(x)} P\left(\frac{e^{-\theta \Delta} \widetilde{U}_{n}}{\widetilde{X}_{n-1}} \geq x\right)-1\right| \\
& =\sup _{2 M \leq x \leq \rho \beta_{n, \Delta}}\left|\frac{F_{\mathbb{C}}(x)-F_{\mathbb{C}}\left(A_{n}^{-1}\left(x-B_{n}\right)\right)}{1-F_{\mathbb{C}}(x)}\right| \\
& \leq \frac{x^{2}}{(1-\epsilon)\left(1+2 M^{2}\right)} O\left(n \Delta e^{-\theta n \Delta}\right) .
\end{aligned}
$$

Therefore

$$
\lim _{\Delta \rightarrow 0, n \rightarrow \infty} \sup _{2 M \leq x \leq \rho \beta_{n, \Delta}}\left|\frac{1}{1-F_{\mathbb{C}}(x)} P\left(\frac{e^{-\theta \Delta} \widetilde{U}_{n}}{\widetilde{X}_{n-1}} \geq x\right)-1\right|=0 .
$$

Furthermore, it is easy to obtain that

$$
\lim _{\Delta \rightarrow 0, n \rightarrow \infty} \sup _{0 \leq x \leq 2 M}\left|\frac{1}{1-F_{\mathbb{C}}(x)} P\left(\frac{e^{-\theta \Delta} \widetilde{U}_{n}}{\widetilde{X}_{n-1}} \geq x\right)-1\right|=0 .
$$

Together with (4.11), we can prove (4.8). Moreover, (4.9) can be shown similarly, and the details are omitted here.

In the remainder of this subsection, we will show that $R_{1 n}, R_{2 n}$ and $\widetilde{R}_{3 n}$ defined in (4.5) and (4.7), are negligible in the sense of Cramér-type moderate deviations. To do this, we need the following deviation inequality for the multiple Wiener-Itô integrals ([34], Theorem 2 or [35], Theorem 4.1).

Lemma 4.3. For any symmetric function $f \in L^{2}\left([0, T]^{n}\right)$ and $x>0$, we have

$$
P\left(\left|I_{n}(f)\right|>x\right) \leq C \exp \left\{-\frac{1}{2}\left(\frac{x}{\sqrt{n !}\|f\|_{L^{2}\left([0, T]^{n}\right)}}\right)^{\frac{2}{n}}\right\},
$$

where $I_{n}(f)$ is the $n$-th Wiener-Itô integral of $f$ with respect to the Wiener process ([36], [38]), and the constant $C>0$ depends only on the multiplicity $n$ of the integral.

Lemma 4.4. Let $R_{1 n}$ be defined in (4.5). For $\beta_{n, \Delta}$ defined by (1.5), we have

$$
\lim _{\Delta \rightarrow 0, n \rightarrow \infty} \beta_{n, \Delta} P\left(\left|R_{1 n}\right| \geq \frac{1}{\beta_{n, \Delta} \log \beta_{n, \Delta}}\right)=0 .
$$


Proof. For $R_{1 n}=\frac{e^{-\theta \Delta} \widetilde{U}_{n}}{\tilde{X}_{n-1}} \cdot \frac{e^{-\theta n \Delta} \epsilon_{n}^{\Delta}(\theta)}{\tilde{X}_{n-1}}$, we can wirte that

$$
\begin{aligned}
& P\left(\left|R_{1 n}\right| \geq \frac{1}{\beta_{n, \Delta} \log \beta_{n, \Delta}}\right) \\
& \leq P\left(\left|\frac{e^{-\theta \Delta} \widetilde{U}_{n}}{\widetilde{X}_{n-1}}\right| \geq \frac{\beta_{n, \Delta}}{\epsilon}\right)+P\left(\left|\widetilde{X}_{n-1}\right| \leq \frac{\epsilon}{\beta_{n, \Delta}}\right) \\
& \quad+P\left(\left|e^{-\theta n \Delta} \epsilon_{n}^{\Delta}(\theta)\right| \geq \frac{\epsilon^{2}}{\beta_{n, \Delta}^{3} \log \beta_{n, \Delta}}\right) .
\end{aligned}
$$

Using (3.8) and Lemma 4.2, we have

$$
\lim _{\epsilon \rightarrow 0} \lim _{\Delta \rightarrow 0, n \rightarrow \infty} \beta_{n, \Delta} P\left(\left|\frac{e^{-\theta \Delta} \widetilde{U}_{n}}{\widetilde{X}_{n-1}}\right| \geq \frac{\beta_{n, \Delta}}{\epsilon}\right)=0 .
$$

Note that $\widetilde{X}_{n-1} \sim N\left(0, \frac{1}{2 \theta}\left(1-e^{-2 \theta(n-1) \Delta}\right)\right)$. Then

$$
P\left(\left|\widetilde{X}_{n-1}\right| \leq \frac{\epsilon}{\beta_{n, \Delta}}\right) \leq \frac{C \epsilon}{\beta_{n, \Delta}}
$$

which implies that

$$
\lim _{\epsilon \rightarrow 0} \lim _{\epsilon \rightarrow 0, n \rightarrow \infty} \beta_{n, \Delta} P\left(\left|\widetilde{X}_{n-1}\right| \leq \frac{\epsilon}{\beta_{n, \Delta}}\right)=0 .
$$

From the fact $e^{-\theta n \Delta} \epsilon_{n}^{\Delta}(\theta) \sim N\left(0, \frac{1}{2 \theta} e^{-2 \theta n \Delta}\left(e^{2 \theta \Delta}-1\right)\right)$, it follows that

$$
P\left(\left|e^{-\theta n \Delta} \epsilon_{n}^{\Delta}(\theta)\right| \geq \frac{\epsilon^{2}}{\beta_{n, \Delta}^{3} \log \beta_{n, \Delta}}\right) \leq 2 \exp \left\{-\frac{C \epsilon^{4} e^{2 \theta n \Delta}}{\Delta \beta_{n, \Delta}^{6}\left(\log \beta_{n, \Delta}\right)^{2}}\right\},
$$

which implies immediately

$$
\lim _{\Delta \rightarrow 0, n \rightarrow \infty} \beta_{n, \Delta} P\left(\left|e^{-\theta n \Delta} \epsilon_{n}^{\Delta}(\theta)\right| \geq \frac{\epsilon^{2}}{\beta_{n, \Delta}^{3} \log \beta_{n, \Delta}}\right)=0 .
$$

Combing (4.13)-(4.15), we can obtain that

$$
\lim _{\Delta \rightarrow 0, n \rightarrow \infty} \beta_{n, \Delta} P\left(\left|R_{1 n}\right| \geq \frac{1}{\beta_{n, \Delta} \log \beta_{n, \Delta}}\right)=0 .
$$

Lemma 4.5. Let $R_{2 n}$ and $\widetilde{R}_{3 n}$ be defined in (4.7). For $\beta_{n, \Delta}$ defined by (1.5), we have

$$
\lim _{\Delta \rightarrow 0, n \rightarrow \infty} \beta_{n, \Delta} P\left(\left|R_{2 n}\right| \vee\left|\widetilde{R}_{3 n}\right| \geq \frac{1}{\beta_{n, \Delta} \log \beta_{n, \Delta}}\right)=0 .
$$


Proof. To begin with, we establish the deviation inequalities for $r_{1 n}$ and $r_{2 n}$ defined in (4.3) and (4.4). In fact, by (4.3), $r_{1 n}$ can be rewritten as

$$
\begin{aligned}
r_{1 n}= & e^{-3 \theta \Delta} \sum_{i=1}^{n} e^{-\theta(n-i) \Delta} \epsilon_{i}^{\Delta}(\theta)\left(\widetilde{X}_{i-1}-\widetilde{X}_{i}\right) \\
& +e^{-3 \theta \Delta} \sum_{i=1}^{n} e^{-\theta(n-i) \Delta} \epsilon_{i}^{\Delta}(\theta)\left(\widetilde{X}_{i}-\widetilde{X}_{n}\right):=r_{11 n}+r_{12 n} .
\end{aligned}
$$

Straightforward calculation gives that

$$
E r_{11 n}=O\left(n \Delta e^{-\theta n \Delta}\right), \quad \operatorname{Var}\left(r_{11 n}\right)=O\left(n \Delta^{2} e^{-2 \theta n \Delta}\right)
$$

and

$$
E r_{12 n}=0, \quad \operatorname{Var}\left(r_{12 n}\right)=O\left(n \Delta e^{-2 \theta n \Delta}\right) .
$$

Note that $r_{1 n}-E r_{11 n}$ is a second order Wiener-Itô integral. Using Lemma 4.3, we obtain for any $r>0$

$$
P\left(\left|r_{1 n}-E r_{11 n}\right| \geq r\right) \leq C_{1} \exp \left\{-C_{2} r(n \Delta)^{-1 / 2} e^{\theta n \Delta}\right\} .
$$

Moreover, we also

$$
E r_{2 n}=O\left(n \Delta e^{-2 \theta n \Delta}\right), \quad \operatorname{Var}\left(r_{2 n}\right)=O\left(e^{-2 \theta n \Delta}\right)
$$

Since $r_{2 n}-E r_{2 n}$ is a second order Wiener-Itô integral, using Lemma 4.3 again, we obtain for any $r>0$

$$
P\left(\left|r_{2 n}-E r_{2 n}\right| \geq r\right) \leq C_{1} \exp \left\{-C_{2} r e^{\theta n \Delta}\right\} .
$$

Secondly, for $R_{2 n}=\frac{r_{1 n}}{e^{-2 \theta \Delta} \tilde{X}_{n-1}^{2}-r_{2 n}}$ and $R_{3 n}=\frac{r_{2 n}}{e^{-2 \theta \Delta} \tilde{X}_{n-1}^{2}-r_{2 n}}$, we have for any $\epsilon>0$

$$
\begin{aligned}
& P\left(\left|R_{2 n}\right| \geq \frac{1}{\beta_{n, \Delta} \log \beta_{n, \Delta}}\right) \\
& \leq P\left(\left|e^{-2 \theta \Delta} \widetilde{X}_{n-1}^{2}\right| \leq \frac{2 \epsilon}{\beta_{n, \Delta}^{2}}\right)+P\left(\left|r_{2 n}\right| \geq \frac{\epsilon}{\beta_{n, \Delta}^{2}}\right)+P\left(\left|r_{1 n}\right| \geq \frac{\epsilon}{\beta_{n, \Delta}^{3} \log \beta_{n, \Delta}}\right)
\end{aligned}
$$

and

$$
\begin{aligned}
& P\left(\left|R_{3 n}\right| \geq \frac{\epsilon}{\beta_{n, \Delta}^{2} \log \beta_{n, \Delta}}\right) \\
& \leq P\left(\left|e^{-2 \theta \Delta} \widetilde{X}_{n-1}^{2}\right| \leq \frac{2 \delta}{\beta_{n, \Delta}^{2}}\right)+P\left(\left|r_{2 n}\right| \geq \frac{\delta}{\beta_{n, \Delta}^{2}}\right)+P\left(\left|r_{2 n}\right| \geq \frac{\epsilon \delta}{\beta_{n, \Delta}^{4} \log \beta_{n, \Delta}}\right) .
\end{aligned}
$$

From (4.14), it follows that

$$
\lim _{\delta \rightarrow 0} \lim _{\Delta \rightarrow 0, n \rightarrow \infty} \beta_{n, \Delta} P\left(\left|e^{-2 \theta \Delta} \tilde{X}_{n-1}^{2}\right| \leq \frac{2 \delta}{\beta_{n, \Delta}^{2}}\right)=0 .
$$


Moreover, by (4.17)-(4.21) and the condition (1.5) for $\beta_{n, \Delta}$,

$$
\begin{aligned}
& \lim _{\Delta \rightarrow 0, n \rightarrow \infty} \beta_{n, \Delta} \max \left\{P\left(\left|r_{1 n}\right| \geq \frac{\epsilon}{\beta_{n, \Delta}^{3} \log \beta_{n, \Delta}}\right), P\left(\left|r_{2 n}\right| \geq \frac{\epsilon \delta}{\beta_{n, \Delta}^{4} \log \beta_{n, \Delta}}\right)\right\} \\
& =0 .
\end{aligned}
$$

Therefore, we have

$$
\begin{aligned}
& \lim _{\Delta \rightarrow 0, n \rightarrow \infty} \beta_{n, \Delta} \max \left\{P\left(\left|R_{2 n}\right| \geq \frac{1}{\beta_{n, \Delta} \log \beta_{n, \Delta}}\right), P\left(\left|R_{3 n}\right| \geq \frac{\epsilon}{\beta_{n, \Delta}^{2} \log \beta_{n, \Delta}}\right)\right\} \\
& =0 .
\end{aligned}
$$

Finally, we are ready to deal with the term $\widetilde{R}_{3 n}$. In fact, for any $\epsilon>0$

$$
\begin{aligned}
& P\left(\left|\widetilde{R}_{3 n}\right| \geq \frac{1}{\beta_{n, \Delta} \log \beta_{n, \Delta}}\right) \\
& \leq P\left(\left|\frac{e^{-n \Delta} \widetilde{U}_{n}}{\widetilde{X}_{n-1}}\right| \geq \frac{\beta_{n, \Delta}}{\epsilon}\right)+P\left(\left|R_{1 n}\right| \geq \frac{\beta_{n, \Delta}}{\epsilon}\right)+P\left(\left|R_{3 n}\right| \geq \frac{2 \epsilon}{\beta_{n, \Delta}^{2} \log \beta_{n, \Delta}}\right) .
\end{aligned}
$$

Applying (4.12), (4.13) and (4.22), we have

$$
\lim _{\Delta \rightarrow 0, n \rightarrow \infty} \beta_{n, \Delta} P\left(\left|\widetilde{R}_{3 n}\right| \geq \frac{1}{\beta_{n, \Delta} \log \beta_{n, \Delta}}\right)=0 .
$$

Together with (4.22) and (4.23), we can complete the proof of this lemma.

Proof of Proposition 3.1. By (3.8), for any $\epsilon>0$, there exists some positive constant $M$ such that

$$
\sup _{x \geq M}\left|\pi x\left(1-F_{\mathbb{C}}(x)\right)-1\right| \leq \epsilon .
$$

Now, for each $\rho>0$ and $2 M \leq x \leq \rho \beta_{n, \Delta}$, we have by (4.6)

$$
\begin{aligned}
& P\left(\frac{e^{-n \Delta} \widetilde{U}_{n}}{\widetilde{X}_{n-1}} \geq x+\frac{1}{\beta_{n, \Delta} \log \beta_{n, \Delta}}\right)-P\left(\left|R_{1 n}+R_{2 n}+\widetilde{R}_{3 n}\right| \geq \frac{1}{\beta_{n, \Delta} \log \beta_{n, \Delta}}\right) \\
\leq & P\left(\frac{e^{-\theta(n+2) \Delta} U_{n}}{\left(1-e^{-2 \theta \Delta}\right) e^{-2 \theta n \Delta} V_{n}} \geq x\right) \\
\leq & P\left(\frac{e^{-n \Delta} \widetilde{U}_{n}}{\widetilde{X}_{n-1}} \geq x-\frac{1}{\beta_{n, \Delta} \log \beta_{n, \Delta}}\right)+P\left(\left|R_{1 n}+R_{2 n}+\widetilde{R}_{3 n}\right| \geq \frac{1}{\beta_{n, \Delta} \log \beta_{n, \Delta}}\right) .
\end{aligned}
$$

Firstly, by using Lemma 4.4, Lemma 4.5, and (4.24), we have

$$
\lim _{\Delta \rightarrow 0, n \rightarrow \infty} \sup _{M \leq x \leq \rho \beta_{n, \Delta}} \frac{P\left(\left|R_{1 n}+R_{2 n}+\widetilde{R}_{3 n}\right| \geq \frac{1}{\beta_{n, \Delta} \log \beta_{n, \Delta}}\right)}{1-F_{\mathbb{C}}(x)}=0 .
$$


Secondly, we can write that

$$
\begin{aligned}
& \sup _{2 M \leq x \leq \rho \beta_{n, \Delta}}\left|\frac{P\left(\frac{e^{-n \Delta} \widetilde{U}_{n}}{\widetilde{X}_{n-1}} \geq x \pm \frac{1}{\beta_{n, \Delta} \log \beta_{n, \Delta}}\right)}{1-F_{\mathbb{C}}(x)}-1\right| \\
& \leq \sup _{2 M \leq x \leq \rho \beta_{n, \Delta}}\left|\frac{1-F_{\mathbb{C}}\left(x \pm \frac{1}{\beta_{n, \Delta} \log \beta_{n, \Delta}}\right)}{1-F_{\mathbb{C}}(x)}-1\right| \\
& +\sup _{2 M \leq x \leq \rho \beta_{n, \Delta}}\left|\frac{1-F_{\mathbb{C}}\left(x \pm \frac{1}{\beta_{n, \Delta} \log \beta_{n, \Delta}}\right)}{1-F_{\mathbb{C}}(x)}\right|\left|\frac{P\left(\frac{e^{-n \Delta} \widetilde{U}_{n}}{\widetilde{X}_{n-1}} \geq x \pm \frac{1}{\beta_{n, \Delta} \log \beta_{n, \Delta}}\right)}{1-F_{\mathbb{C}}\left(x \pm \frac{1}{\beta_{n, \Delta} \log \beta_{n, \Delta}}\right)}-1\right| .
\end{aligned}
$$

Together with Lemma 4.2 and (3.12), we can obtain that

$$
\lim _{\Delta \rightarrow 0, n \rightarrow \infty} \sup _{2 M \leq x \leq \rho \beta_{n, \Delta}}\left|\frac{P\left(\frac{e^{-n \Delta} \widetilde{U}_{n}}{\widetilde{X}_{n-1}} \geq x \pm \frac{1}{\beta_{n, \Delta} \log \beta_{n, \Delta}}\right)}{1-F_{\mathbb{C}}(x)}-1\right|=0 .
$$

Therefore, by (4.25)-(4.27), it holds that

$$
\lim _{\Delta \rightarrow 0, n \rightarrow \infty} \sup _{2 M \leq x \leq \rho \beta_{n, \Delta}}\left|\frac{1}{1-F_{\mathbb{C}}(x)} P\left(\frac{e^{-\theta(n+2) \Delta} U_{n}}{\left(1-e^{-2 \theta \Delta}\right) e^{-2 \theta n \Delta} V_{n}} \geq x\right)-1\right|=0 .
$$

Moreover, since the function $1-F_{\mathbb{C}}(x)$ is uniformly continuous, the standard discretized approximation argument shows that

$$
\lim _{\Delta \rightarrow 0, n \rightarrow \infty} \sup _{x \in \mathbb{R}}\left|P\left(\frac{e^{-\theta(n+2) \Delta} U_{n}}{\left(1-e^{-2 \theta \Delta}\right) e^{-2 \theta n \Delta} V_{n}} \geq x\right)-\left(1-F_{\mathbb{C}}(x)\right)\right|=0 .
$$

Therefore, it follows that

$$
\lim _{\Delta \rightarrow 0, n \rightarrow \infty} \sup _{0 \leq x \leq 2 M}\left|\frac{1}{1-F_{\mathbb{C}}(x)} P\left(\frac{e^{-\theta(n+2) \Delta} U_{n}}{\left(1-e^{-2 \theta \Delta}\right) e^{-2 \theta n \Delta} V_{n}} \geq x\right)-1\right|=0 .
$$

Finally, (3.5) can be obtained immediately by (4.28) and (4.29). Using the same procedure, we can prove (3.6), and the details are omitted here.

\subsection{Proof of Proposition 3.2}

In this part, we will prove the Cramér-type moderate deviation for $\frac{(n \Delta)^{-1 / 2} e^{-\theta \Delta} U_{n}}{\sqrt{-2 \theta} n^{-1} V_{n}}$, where $U_{n}, V_{n}$ are defined by (3.4). Firstly, we will represent $U_{n}$ by second order Wiener-Itô integral, while its forth moment can be estimated explicitly. Then, we can get the Cramér-type moderate deviation for $U_{n}$ by using the results in Schulte and Thäle ([41]). Secondly, for the term $V_{n}$, we will show that it is exponential equivalent to its asymptotic expectation in the sense of moderate deviation. 
Lemma 4.6. For $s, t \in[0, n \Delta]$, define

$$
\begin{aligned}
\varphi_{n, \Delta}(s, t)=\sum_{i=1}^{n} e^{(2 i-1) \Delta \theta} e^{-\theta(t+s)}\left(\mathbb{I}_{[(i-1) \Delta, i \Delta]}(t) \cdot \mathbb{I}_{[0,(i-1) \Delta]}(s)\right. & \\
& \left.+\mathbb{I}_{[(i-1) \Delta, i \Delta]}(s) \cdot \mathbb{I}_{[0,(i-1) \Delta]}(t)\right),
\end{aligned}
$$

where $\mathbb{I}_{A}(\cdot)$ is the indicative function of $A$. Then

$$
U_{n}=\frac{1}{2} I_{2}\left(\varphi_{n, \Delta}\right)
$$

and

$$
E\left(\left\|D I_{2}(\varphi(s, t))\right\|_{\mathbb{H}}^{2}-E \| D I_{2}\left(\varphi(s, t) \|_{\mathbb{H}}^{2}\right)^{2}=O(n \Delta),\right.
$$

where $I_{2}(\cdot)$ is the second order Wiener-Itô integral with respect to Brownian motion, $D$ is the Malliavin derivative operator and $\mathbb{H}$ is a Hilbert space associated with Brownian motion (see Nualart ([38])).

Proof. By straightforward calculation, we have

$$
\begin{aligned}
U_{n}= & \sum_{i=1}^{n} e^{(2 i-1) \theta \Delta} \int_{0}^{n \Delta} e^{-\theta s} \mathbb{I}_{[(i-1) \Delta, i \Delta]}(s) d W_{s} \int_{0}^{n \Delta} e^{-\theta s} \mathbb{I}_{[0,(i-1) \Delta]}(s) d W_{s} \\
= & \sum_{i=1}^{n} e^{(2 i-1) \theta \Delta}\left(\int_{0}^{n \Delta} \int_{0}^{s} e^{-\theta t} \mathbb{I}_{[(i-1) \Delta, i \Delta]}(t) \cdot e^{-\theta s} \mathbb{I}_{[0,(i-1) \Delta]}(s) d W_{t} d W_{s}\right. \\
& \left.+\int_{0}^{n \Delta} \int_{0}^{s} e^{-\theta t} \mathbb{I}_{[0,(i-1) \Delta]}(t) \cdot e^{-\theta s} \mathbb{I}_{[(i-1) \Delta, i \Delta]}(s) d W_{t} d W_{s}\right) \\
= & \frac{1}{2} I_{2}\left(\varphi_{n, \Delta}\right) .
\end{aligned}
$$

Using (4.30), we have for $s \in[0, n \Delta]$

$$
\begin{aligned}
D_{s}\left(I_{2}\left(\varphi_{n, \Delta}\right)\right)= & 2 \sum_{i=1}^{n} e^{(2 i-1) \theta \Delta} \mathbb{I}_{[0,(i-1) \Delta]}(s) \int_{(i-1) \Delta}^{i \Delta} e^{-\theta(t+s)} d W_{t} \\
& +2 \sum_{i=1}^{n} e^{(2 i-1) \theta \Delta} \mathbb{I}_{[(i-1) \Delta, i \Delta]}(s) \int_{0}^{(i-1) \Delta} e^{-\theta(t+s)} d W_{t} \\
= & 2 Y_{s}+2 \tilde{Y}_{s},
\end{aligned}
$$

which implies that

$$
\begin{aligned}
\frac{1}{4} \| D I_{2}\left(\varphi(s, t) \|_{\mathbb{H}}^{2}\right. & =\int_{0}^{n \Delta} Y_{t}^{2} d t+\int_{0}^{n \Delta} \tilde{Y}_{t}^{2} d t+2 \int_{0}^{n \Delta} Y_{t} \tilde{Y}_{t} d t \\
& =A_{n, \Delta}^{(1)}+A_{n, \Delta}^{(2)}+A_{n, \Delta}^{(3)} .
\end{aligned}
$$


Firstly, for $A_{n, \Delta}^{(1)}$, we have

$$
\begin{aligned}
Y_{t}^{2}= & \sum_{i=1}^{n} e^{2(i-1) \Delta \theta} e^{-2 \theta t} \mathbb{I}_{[0,(i-1) \Delta]}(t)\left(\epsilon_{i}^{\Delta}(\theta)\right)^{2} \\
& +2 \sum_{i=1}^{n} \sum_{j=1}^{i-1} e^{(i+j-2) \Delta \theta} e^{-2 \theta t} \mathbb{I}_{[0,(j-1) \Delta]}(t) \epsilon_{i}^{\Delta}(\theta) \epsilon_{j}^{\Delta}(\theta),
\end{aligned}
$$

where $\epsilon_{i}^{\Delta}(\theta)=e^{i \Delta \theta} \int_{(i-1) \Delta}^{i \Delta} e^{-\theta s} d W_{s}$. Therefore,

$$
\begin{aligned}
A_{n, \Delta}^{(1)}= & -\frac{1}{2 \theta} \sum_{i=1}^{n}\left(1-e^{2(i-1) \Delta \theta}\right)\left(\epsilon_{i}^{\Delta}(\theta)\right)^{2} \\
& -\frac{1}{\theta} \sum_{i=1}^{n} \sum_{j=1}^{i-1}\left(e^{(i-j) \Delta \theta}-e^{(i+j-2) \Delta \theta}\right) \epsilon_{i}^{\Delta}(\theta) \epsilon_{j}^{\Delta}(\theta) .
\end{aligned}
$$

Then

$$
E A_{n, \Delta}^{(1)}=n \frac{1-e^{2 \theta \Delta}}{4 \theta^{2}}-\frac{1-e^{2 \theta n \Delta}}{4 \theta^{2}}, \quad E\left(A_{n, \Delta}^{(1)}\right)^{2}=\frac{(n \Delta)^{2}}{4 \theta^{2}}-\frac{n \Delta}{4 \theta^{3}}+o(n \Delta),
$$

which implies that

$$
E\left(A_{n, \Delta}^{(1)}-E\left(A_{n, \Delta}^{(1)}\right)\right)^{2}=-\frac{n \Delta}{2 \theta^{3}}+o(n \Delta) .
$$

Secondly, for $A_{n, \Delta}^{(2)}$, it holds that

$$
\tilde{Y}_{t}^{2}=\sum_{i=1}^{n} e^{2(2 i-1) \Delta \theta} e^{-2 \theta t} \mathbb{I}_{[(i-1) \Delta, i \Delta]}(t)\left(\int_{0}^{(i-1) \Delta} e^{-\theta s} d W_{s}\right)^{2}
$$

and

$$
A_{n, \Delta}^{(2)}=-\frac{1}{2 \theta} \sum_{i=1}^{n}\left(e^{2(i-1) \Delta \theta}-e^{2 i \Delta \theta}\right)\left(\int_{0}^{(i-1) \Delta} e^{-\theta s} d W_{s}\right)^{2} .
$$

Then, we can obtain that

$$
E A_{n, \Delta}^{(2)}=n \frac{1-e^{2 \theta \Delta}}{4 \theta^{2}}-\frac{1-e^{2 \theta n \Delta}}{4 \theta^{2}}, \quad E\left(A_{n, \Delta}^{(2)}\right)^{2}=\frac{n^{2} \Delta^{2}}{4 \theta^{2}}-\frac{n \Delta}{4 \theta^{3}}+o(n \Delta),
$$

which implies that

$$
E\left(A_{n, \Delta}^{(2)}-E\left(A_{n, \Delta}^{(2)}\right)\right)^{2}=-\frac{n \Delta}{2 \theta^{3}}+o(n \Delta) .
$$


Finally, for the $A_{n, \Delta}^{(3)}$, we can write that

$$
Y_{t} \tilde{Y}_{t}=\sum_{i=1}^{n} \sum_{j=1}^{i-1} e^{2(i+j-1) \Delta \theta} e^{-2 \theta t} \mathbb{I}_{[(j-1) \Delta, j \Delta]}(t) \int_{0}^{(j-1) \Delta} e^{-\theta s} d W_{s} \int_{(i-1) \Delta}^{i \Delta} e^{-\theta s} d W_{s},
$$

and then

$$
A_{n, \Delta}^{(3)}=-\frac{1}{\theta} \sum_{i=1}^{n} \sum_{j=1}^{i-1}\left(e^{2(i-1) \Delta \theta}-e^{(2 i-1) \Delta \theta}\right) \int_{0}^{(j-1) \Delta} e^{-\theta s} d W_{s} \int_{(i-1) \Delta}^{i \Delta} e^{-\theta s} d W_{s} .
$$

Since

$$
E A_{n, \Delta}^{(3)}=0, \quad E\left(A_{n, \Delta}^{(3)}\right)^{2}=o(n \Delta) .
$$

We can also obtain

$$
E\left(A_{n, \Delta}^{(3)}-E\left(A_{n, \Delta}^{(3)}\right)\right)^{2}=o(n \Delta) .
$$

Together with (4.32)-(4.34), we can complete the proof of this lemma.

Lemma 4.7. Let $\lambda_{n, \Delta}$ defined by (1.16). For each $\rho>0$, uniformly for $0 \leq$ $x \leq \rho \lambda_{n, \Delta}$,

$$
\frac{1}{1-\Phi(x)} P\left( \pm \sqrt{-\frac{2 \theta}{n \Delta}} U_{n} \geq x\right) \rightarrow 1
$$

Proof. Denote by $F_{n, \Delta}=\sqrt{-\frac{2 \theta}{n \Delta}} U_{n}$. By (4.30), we have $F_{n, \Delta}=\sqrt{-\frac{\theta}{2 n \Delta}} I_{2}\left(\varphi_{n, \Delta}\right)$. Then, we can calculate variance of $U_{n}$ as

$$
\begin{aligned}
\operatorname{Var}\left(U_{n}\right) & =\frac{1}{4 \theta^{2}}\left(e^{2 \theta n \Delta}-1+n\left(1-e^{-2 \theta \Delta}\right)\right) \\
& =-\frac{1}{2 \theta} n \Delta-\frac{1}{4 \theta^{2}}-\frac{1}{2} n \Delta^{2}+o\left(n \Delta^{2}\right) .
\end{aligned}
$$

Using Lemma 2 in ([39]), we can obtain

$$
E\left(\left\|D F_{n, \Delta}\right\|_{\mathbb{H}}^{2}-E\left\|D F_{n, \Delta}\right\|_{\mathbb{H}}^{2}\right)^{2}=4 \theta^{2}\left\|\frac{\varphi_{n, \Delta}}{\sqrt{n \Delta}} \otimes_{1} \frac{\varphi_{n, \Delta}}{\sqrt{n \Delta}}\right\|_{\mathbb{H} \otimes 2}^{2} .
$$

From (4.31) in Lemma 4.6, it follows

$$
\left\|\frac{\varphi_{n, \Delta}}{\sqrt{n \Delta}} \otimes_{1} \frac{\varphi_{n, \Delta}}{\sqrt{n \Delta}}\right\|_{\mathbb{H}^{\otimes 2}}^{2}=O\left((n \Delta)^{-1}\right) .
$$

According to Theorem 5 in ([41]) (see also Theorem A.1 in the Appendix A), we have for each $\rho>0$, uniformly for $0 \leq x \leq \rho \lambda_{n, \Delta}$,

$$
\frac{1}{1-\Phi(x)} P\left( \pm \sqrt{-\frac{2 \theta}{n \Delta}} U_{n} \geq x \sqrt{-\frac{2 \theta}{n \Delta} \operatorname{Var}\left(U_{n}\right)}\right) \rightarrow 1 .
$$


Moreover, under the condition that $\lim _{\Delta \rightarrow 0, n \rightarrow \infty} n \Delta^{4}(\log n \Delta)^{6}=0$, we have

$$
\sup _{0 \leq x \leq \rho \lambda_{n, \Delta}}\left|x\left(\sqrt{-\frac{2 \theta}{n \Delta} \operatorname{Var}\left(U_{n}\right)}-1\right)\right| \leq \frac{1}{\lambda_{n, \Delta} \log \lambda_{n, \Delta}} .
$$

By the procedure used in the proof of Theorem 1.5, we have

$$
\lim _{\Delta \rightarrow 0, n \rightarrow \infty} \sup _{0 \leq x \leq \rho \lambda_{n, \Delta}}\left|\frac{1-\Phi\left(x \sqrt{-\frac{2 \theta}{n \Delta} \operatorname{Var}\left(U_{n}\right)}\right)}{1-\Phi(x)}-1\right|=0,
$$

which completes the proof of this lemma.

Lemma 4.8. For $V_{n}$ defined by (3.4), we have

$$
E V_{n}=-\frac{n}{2 \theta}+\frac{1-e^{2 \theta n \Delta}}{2 \theta\left(1-e^{2 \theta \Delta}\right)}
$$

and for any $x>0$

$$
P\left(\frac{1}{n}\left|V_{n}-E V_{n}\right| \geq x\right) \leq C_{1} e^{-C_{2} \sqrt{n \Delta} x}
$$

Proof. Since

$$
V_{n}=\sum_{i=1}^{n} X_{i-1}^{2}=\sum_{i=1}^{n} e^{2(i-1) \Delta \theta}\left(\int_{0}^{(i-1) \Delta} e^{-\theta t} d W_{t}\right)^{2},
$$

we can obtain immediately that

$$
E V_{n}=-\frac{n}{2 \theta}+\frac{1-e^{2 \theta n \Delta}}{2 \theta\left(1-e^{2 \theta \Delta}\right)}, \quad \operatorname{Var}\left(V_{n}\right)=-\frac{n}{2 \theta^{3} \Delta}+o(n / \Delta) .
$$

Note that $V_{n}-E V_{n}$ can be represented as a second order Wiener-Itô integral. Then, using Lemma 4.3, we obtain (4.38).

Proof of Proposition 3.2. For each $\rho>0$ and $0 \leq x \leq \rho \lambda_{n, \Delta}$, we obtain

$$
\begin{aligned}
& \frac{1}{1-\Phi(x)} P\left(\frac{(n \Delta)^{-1 / 2} e^{-\theta \Delta} U_{n}}{\sqrt{-2 \theta} n^{-1} V_{n}} \geq x\right) \\
& \leq \frac{1}{1-\Phi(x)} P\left(\left|n^{-1} V_{n}+\frac{1}{2 \theta}\right| \geq \frac{\lambda_{n, \Delta}^{2} \log n \Delta}{\sqrt{n \Delta}}\right) \\
& \quad+\frac{P\left( \pm \sqrt{-\frac{2 \theta}{n \Delta}} U_{n} \geq x e^{\theta \Delta}\left(1+\frac{2 \theta \lambda_{n, \Delta}^{2} \log n \Delta}{\sqrt{n \Delta}}\right)\right)}{1-\Phi\left(x e^{\theta \Delta}\left(1+\frac{2 \theta \lambda_{n, \Delta}^{2} \log n \Delta}{\sqrt{n \Delta}}\right)\right)}
\end{aligned}
$$




$$
\frac{1-\Phi\left(x e^{\theta \Delta}\left(1+\frac{2 \theta \lambda_{n, \Delta}^{2} \log n \Delta}{\sqrt{n \Delta}}\right)\right)}{1-\Phi(x)} \text {. }
$$

Similarly,

$$
\begin{gathered}
\frac{1}{1-\Phi(x)} P\left(\frac{(n \Delta)^{-1 / 2} e^{-\theta \Delta} U_{n}}{\sqrt{-2 \theta} n^{-1} V_{n}} \geq x\right) \\
\geq-\frac{1}{1-\Phi(x)} P\left(\left|n^{-1} V_{n}+\frac{1}{2 \theta}\right| \geq \frac{\lambda_{n, \Delta}^{2} \log n \Delta}{\sqrt{n \Delta}}\right) \\
+\frac{P\left( \pm \sqrt{-\frac{2 \theta}{n \Delta}} U_{n} \geq x e^{\theta \Delta}\left(1-\frac{2 \theta \lambda_{n, \Delta}^{2} \log n \Delta}{\sqrt{n \Delta}}\right)\right)}{1-\Phi\left(x e^{\theta \Delta}\left(1-\frac{2 \theta \lambda_{n, \Delta}^{2} \log n \Delta}{\sqrt{n \Delta}}\right)\right)} \\
\cdot \frac{1-\Phi\left(x e^{\theta \Delta}\left(1-\frac{2 \theta \lambda_{n, \Delta}^{2} \log n \Delta}{\sqrt{n \Delta}}\right)\right)}{1-\Phi(x)} .
\end{gathered}
$$

From Lemma 4.7, it follows immediately that

$$
\lim _{\Delta \rightarrow 0, n \rightarrow \infty} \sup _{0 \leq x \leq \rho \lambda_{n, \Delta}}\left|\frac{P\left( \pm \sqrt{-\frac{2 \theta}{n \Delta}} U_{n} \geq x e^{\theta \Delta}\left(1 \pm \frac{2 \theta \lambda_{n, \Delta}^{2} \log n \Delta}{\sqrt{n \Delta}}\right)\right)}{1-\Phi\left(x e^{\theta \Delta}\left(1 \pm \frac{2 \theta \lambda_{n, \Delta}^{2} \log n \Delta}{\sqrt{n \Delta}}\right)\right)}-1\right|=0 .
$$

By Lemma 4.8, we have

$$
\lim _{\Delta \rightarrow 0, n \rightarrow \infty} \frac{1}{\lambda_{n, \Delta}^{2}} \log P\left(\left|n^{-1} V_{n}+\frac{1}{2 \theta}\right| \geq \frac{\lambda_{n, \Delta}^{2} \log n \Delta}{\sqrt{n \Delta}}\right)=-\infty,
$$

which implies by (3.27)

$$
\lim _{\Delta \rightarrow 0, n \rightarrow \infty} \sup _{0 \leq x \leq \rho \lambda_{n, \Delta}} \frac{1}{1-\Phi(x)} P\left(\left|n^{-1} V_{n}+\frac{1}{2 \theta}\right| \geq \frac{\lambda_{n, \Delta}^{2} \log n \Delta}{\sqrt{n \Delta}}\right)=0 .
$$

Moreover, by mean value theorem and (3.27), we have such that

$$
\begin{aligned}
& \sup _{0 \leq x \leq \rho \lambda_{n, \Delta}}\left|\frac{1-\Phi\left(x e^{\theta \Delta}\left(1-\frac{2 \theta \lambda_{n, \Delta}^{2} \log n \Delta}{\sqrt{n \Delta}}\right)\right)}{1-\Phi(x)}-1\right| \\
& \leq C_{1}\left(\Delta \lambda_{n, \Delta}^{2} \vee \frac{\lambda_{n, \Delta}^{4} \log n \Delta}{\sqrt{n \Delta}}\right) \exp \left\{-C_{2}\left(\Delta \lambda_{n, \Delta}^{2} \vee \frac{\lambda_{n, \Delta}^{4} \log n \Delta}{\sqrt{n \Delta}}\right)\right\} .
\end{aligned}
$$

Since $\lim _{\Delta \rightarrow 0, n \rightarrow \infty} \Delta \lambda_{n, \Delta}^{2} \leq \lim _{\Delta \rightarrow 0, n \rightarrow \infty}\left(n \Delta^{7}\right)^{1 / 6}$, we can get that

$$
\lim _{\Delta \rightarrow 0, n \rightarrow \infty} \sup _{0 \leq x \leq \rho \lambda_{n, \Delta}}\left|\frac{1-\Phi\left(x e^{\theta \Delta}\left(1-\frac{2 \theta \lambda_{n, \Delta}^{2} \log n \Delta}{\sqrt{n \Delta}}\right)\right)}{1-\Phi(x)}-1\right|=0 .
$$


Finally, together with (4.39)-(4.41), we can completes the proof of this proposition.

\section{Appendix A}

Let $\mathbb{H}$ be a real separable Hilbert space with inner product $<\cdot, \cdot\rangle_{\mathbb{H}}$ and norm $\|\cdot\|_{\mathbb{H}}$. We denote for integer $q \geq 1$ by $\mathbb{H}^{\otimes q}$ the $q$ th tensor power and by $\mathbb{H}^{\odot q}$ the $q$ th symmetric tensor power of $\mathbb{H}$. Moreover, let $\left\{e_{k}, 1 \leq k \leq \operatorname{dim} \mathbb{H}\right\}$ be a complete orthonormal system in $\mathbb{H}$. For integers $p, q \geq 1, f \in \mathbb{H} \odot p, g \in \mathbb{H} \odot q$ and $1 \leq r \leq \min \{p, q\}$, we denote by $f \otimes_{r} g$ the $r$ th contraction of $f$ and $g$ defined as

$$
f \otimes_{r} g=\sum_{i_{1}, \ldots, i_{r}=1}^{\operatorname{dim} \mathbb{H}}<f, e_{i_{1}} \otimes \ldots \otimes e_{i_{r}}>_{\mathbb{H} \otimes r}<g, e_{i_{1}} \otimes \ldots \otimes e_{i_{r}}>_{\mathbb{H} \otimes r} .
$$

Now, let $\left\{h_{n}, n \geq 1\right\}$ be a sequence of elements in $\mathbb{H}^{\odot q}$ for some fixed integer $q \geq 2$. We shall state Theorem 1 and Theorem 5 in Schulte and Thäle ([41]) together as follows.

Theorem A.1. Let $F_{n}:=I_{q}\left(h_{n}\right)$ satisfying

$$
\operatorname{Var}\left(F_{n}\right)=q !\left\|h_{n}\right\|_{\mathbb{H} \otimes q}^{2}=q !
$$

and

$$
\lim _{n \rightarrow \infty} K_{n}=\lim _{\Delta \rightarrow 0, n \rightarrow \infty} \max _{r=1, \cdots, q-1}\left\|h_{n} \otimes_{r} h_{n}\right\|_{\mathbb{H} \otimes 2(q-r)}=0
$$

Define $\triangle_{n}=\left(q^{3 q / 2} K_{n}^{\alpha(q)}\right)^{-1}$, with with

$$
\alpha(q):=\frac{q+2}{3 q+2} \quad(q \text { even }), \quad \alpha(q):=\frac{q^{2}-q-1}{q(3 q-5)} \quad(q \text { odd }) .
$$

Then, there exist constants $c_{0}, c_{1}, c_{2}>0$ depending only on $q$ such tat for $\triangle_{n} \geq$ $c_{0}$ and $0 \leq x \leq c_{1} \triangle_{n}^{1 /(q-1)}$, such that

$$
\left|\log \frac{P\left( \pm F_{n} / \sqrt{q !} \geq x\right)}{1-\Phi(x)}\right| \leq c_{2} \frac{(1+x / \sqrt{q !})^{3}}{\triangle_{n}^{1 /(q-1)}} .
$$

\section{Acknowledgements}

We would like to express our greatest gratitude to $\mathrm{AE}$ for the careful reading and insightful comments (especially the definition of estimator (1.2), Theorem 1.1 and Theorem 1.4), which surely lead to an improved presentation of this paper. 


\section{References}

[1] Alazemi, F., Douissi, S. and Es-Sebaiy, K. (2019). Berry-Esseen bounds and ASCLTs for drift parameter estimator of mixed fractional Ornstein-Uhlenbeck process with discrete observations. Theory of Probability and its Applications, 64 401-420. MR3988271

[2] Azmoodeh, E. and Morlanes, J. (2015). Drift parameter estimation for fractional Ornstein-Uhlenbeck process of the second kind. Statistics, 49 1-18. MR3304364

[3] Barboza, L. A. and Viens, F. G. (2017). Parameter estimation of Gaussian stationary processes using the generalized method of moments. Electronic Journal of Statistics, 11 401-439. MR3611508

[4] Bercu, B. and Rounult, A. (2002). Sharp large deviations for the Ornstein-Uhlenbeck process. Theory of Probability and its Application, 46 1-19. MR1968706

[5] Bercu, B., Coutin, L. and Savy, N. (2012). Sharp large deviations for the non-stationary Ornstein-Uhlenbeck process. Stochastic Processes and their Applications, 122 3393-3424. MR2956110

[6] Bercu, B. and Richou, A. (2015). Large deviations for the OrnsteinUhlenbeck process with shift. Advance in Applied Probability, 47 880-901. MR3406612

[7] Bercu, B. and Richou, A. (2017). Large deviations for the OrnsteinUhlenbeck process without tears. Statistics and Probability Letters, 123 45-55. MR3598618

[8] Chronopoulou, A. and Viens, F. G. (2012). Estimation and pricing under long-memory stochastic volatility. Annals of Finance, 8(2-3) 379403. MR2922802

[9] Comte, F. and Renault, E. (1998). Long memory in continuoustime stochastic volatility models. Mathematical Finance, 8(4) 291-323. MR1645101

[10] Dietz, H. M. and Kutoyants, Yu. A. (2003). Parameter estimation for some non-recurrent solutions of SDE. Statist. Decisions, 21 29-45. MR1985650

[11] Douissi, S., Es-Sebaiy, K. and Viens, F. G. (2019). Berry-Esséen bounds for parameter estimation of general Gaussian processes. ALEA, 16 633-664. MR3949273

[12] El Onsy, B., Es-Sebaiy, K. and Viens, F. G. (2017). Parameter estimation for a partially observed Ornstein-Uhlenbeck process with long-memory noise, Stochastics, 89 431-468. MR3590429

[13] El Onsy, B., Es-Sebaiy, K. and Ndiaye, D. (2018). Parameter estimation for discretely observed non-ergodic fractional Ornstein-Uhlenbeck processes of the second kind. Brazilian Journal of Probability and Statistics, 32 545-558. MR3812381

[14] Es-Sebaiy, K. (2013) Berry-Esséen bounds for the least squares estimator for discretely observed fractional Ornstein-Uhlenbeck processes. Statistics and Probability Letters, 83 2372-2385. MR3093828 
[15] Es-Sebaiy, K. and Viens, F. G. (2019). Optimal rates for parameter estimation of stationary Gaussian processes. Stochastic Processes and their Applications, 129 3018-3054. MR3985554

[16] Florens-Landais, D. and Pham, H. (1999). Large deviations in estimate of an Ornstein-Uhlenbeck model. Journal of Applied Probability, 36 60-77. MR1699608

[17] GAO, F. Q. and JiAnG, H. (2009). Deviation inequalities and moderate deviations for estimators of parameters in an Ornstein-Uhlenbeck process with linear drift. Electronic Communication in Probability, 14 210-223. MR2507750

[18] GaO, F. Q. and Jiang, H. (2017). Deviation inequalities for quadratic Wiener functionals and moderate deviations for parameter estimators. Science China Mathematics, 60 1181-1196. MR3665795

[19] Gao, F. Q., Xiong, J. and Zhao, X. Q. (2018). Moderate deviations and nonparametric inference for monotone functions, The Annals of Statistics, 46 1225-1254. MR3798002

[20] Guillin, A. and Liptser, R. (2006). Examples of moderate deviation principles for diffusion processes. Discrete and Continuous Dynamical Systems, Series B, 6 77-102. MR2223909

[21] Gobet, E. (2002). LAN property for ergodic diffusions with discrete observations. Annales de lInstitut Henri Poincarté Probabilités et Statistiques, 38(5) 711-737. MR1931584

[22] Hu, Y. and Nualart, D. (2010) Parameter estimation for fractional Ornstein-Uhlenbeck processes. Statistics and Probability Letters, 801030 1038. MR2638974

[23] Hu, Y., Nualart, D. and Zhou, H. J. (2019). Parameter estimation for fractional Ornstein-Uhlenbeck processes of general Hurst parameter. Statistical Inference for Stochastic Processes, 22 111-142. MR3918739

[24] Hu, Y. and Song, J. (2013). Parameter estimation for fractional OrnsteinUhlenbeck processes with discrete observations. In Malliavin calculus and stochastic analysis. A Festschrift in honor of David Nualart. New York, NY: Springer 427-442. MR3070455

[25] JACOD, J. (2006). Parametric inference for discretely observed non-ergodic diffusions. Bernoulli, 12(3) 383-401. MR2232724

[26] JiAng, H., LiU, J. F. and Wang, S. C. (2019). Self-normalized asymptotic properties for the parameter estimation in fractional Ornstein-Uhlenbeck process. Stochastics and Dynamics, 19(3) 1950018. MR3956358

[27] JiAng, H. and Zhang, N. (2020). Cramér-type moderate deviations for statistics in the non-stationary Ornstein-Uhlenbeck process. Stochastics, 92(3) 478-496. MR4085761

[28] KASONGA, R. A. (1988). The consistency of a nonlinear least squares estimator from diffusion processes. Stochastic Processes and their Applications, 30 263-275. MR0978358

[29] Kasonga, R. A. (1990). Parameter estimation by deterministic approximation of a solution of a stochastic differential equation. Comm. Statist. Stochastic Models, 6 59-67. MR1047103 
[30] Kessler, M. (1997). Estimation of an ergodic diffusion from discrete observations. Scandinavian Journal of Statistics, 24, 211-229. MR1455868

[31] Kubilius, K., Mishura, Y., Ralchenko, K. and Seleznjev, O. (2015). Consistency of the drift parameter estimator for the discretized fractional Ornstein-Uhlenbeck process with Hurst index $H \in\left(0, \frac{1}{2}\right)$. Electronic Journal of Statistics, 9 1799-1825. MR3391120

[32] Kukush, A., Mishura, Y. and Ralchenko, K. (2017). Hypothesis testing of the drift parameter sign for fractional Ornstein-Uhlenbeck process. Electronic Journal of Statistics, 11 385-400. MR3608678

[33] Kutoyants, Y. A. (2004). Statistical Inference for Ergodic Diffusion Processes. Springer, London. MR2144185

[34] Major, P. (2007). On a multivariate version of Bernsteins inequality. Electronic Journal of Probability, 12 966-988. MR2336595

[35] Major, P. (2005). Tail behavior of multiple integrals and U-statistics. Probability Surveys, 2 448-505. MR2203678

[36] Nourdin, I., and Peccati, G. (2012). Normal Approximations with Malliavin Calculus: From Stein's Method to Universality, Cambridge University Press, Cambridge. MR2962301

[37] Nourdin, I. and Tran, T. (2019). Statistical inference for Vasicek-type model driven by Hermite processes. Stochastic Processes and their Applications, 129(10) 3774-3791. MR3997661

[38] Nualart, D. and Nualart, E. (2018). Introduction to Malliavin Calculus, Cambridge University Press, Cambridge. MR3838464

[39] Nualart, D. and Ortiz-Latorre, S. (2008). Central limit theorems for multiple stochastic integrals and Malliavin calculus. Stochastic Processes and their Applications, 118 614-628. MR2394845

[40] Prakasa Rao, B. L. S. (1999). Statistical Inference for Diffusion Type Processes, Edward Arnold/Oxford University Press, London/New York. MR1717690

[41] Schulte, M. and ThäLe, C. (2016). Cumulants on Wiener chaos: Moderate deviations and the fourth moment theorem. Journal of Functional Analysis, 270 2223-2248. MR3460239

[42] Shimizu, Y. (2009). Notes on drift estimation for non-recurrent OrnsteinUhlenbeck processes from sampled data. Statistics and Probability Letters, 79(20) 2200-2207. MR2572052

[43] Shimizu, Y. (2012). Local asymptotic mixed normality for discretely observed non-recurrent Ornstein-Uhlenbeck processes. Annals of the Institute of Statistical Mathematics, 64 193-211. MR2878923

[44] Tudor, C. A. and Viens, F. G. (2007). Statistical aspects of the fractional stochastic calculus. The Annals of Statistics, 35(3) 1183-1212. MR2341703

[45] VASICEK, O. (1977). An equilibrium characterization of the term structure. Journal of Financial Economics, 5(2) 177-188.

[46] Xiao, W., Zhang, W. and Xu, W. (2011). Parameter estimation for fractional Ornstein-Uhlenbeck processes at discrete observation. Appl. Math. Modelling 35 4196-4207. MR2801946 
[47] Xiao, W. and Yu, J. (2019). Asymptotic theory for estimating drift parameters in the fractional Vasicek model. Econometric Theory, 35 198-231. MR3904176

[48] ZANG, Q. P. and ZhAng, L. X. (2016). A general lower bound of parameter estimation for reflected Ornstein-Uhlenbeck processes. Journal of Applied Probability, 53 22-32. MR3540777

[49] ZANG, Q. P. and ZhANG, L. X. (2019). Asymptotic behaviour of the trajectory fitting estimator for reflected Ornstein-Uhlenbeck processes. Journal of Theoretical Probability, 32 183-201. MR3908911 